\title{
NORME E RISULTATI NELL'USO DEL COMPENSATORE A CORRENTE ALTERNATA «BICARAM )
}

\author{
A. Belluigi
}

1. Premessa. - Con "Accoppiamenti resistivi di due bobine indullive nella prospezione eleltrica a corrente alternata del sottosuolo" ('), iniziai nel 1942 uno studio generale su tali accoppiamenti, deducendo i due casi capacitivi circuitali possibili, con capacità in parallelo o in serie (Bicaram), e come caso particolare $(c=\infty)$ il compensatore Turam.

L'argomento venne da me ripreso e sviluppato quasi subito dopo (1943) in un'ampia Memoria apparsa nel Bollettino dell'Ufficio Geologico d'Ttalia ( $\left.{ }^{1}\right)$.

Nell'ultima parte di questa definii un nuovo strumento di misura a c.a. (corrente alternata) Bicaram, da preferirsi, per varie ragioni, ai precedenti in uso, (un accoppiamento resistivo-capacitivo di 2 bobine induttive con esperimenti di Laboratorio confermanti la teoria data.

Nel 1944 apparve una mia prima Nola sull's Impiego del compensatore a c.a. a bobine bilanciate, nelle misure in scala ridotta " $\left({ }^{1}\right)$.

Col titolo "Bicaram" (Nuova apparecchiatura e.m., eleltromagnetica, a sensibiliti variabile) ( ${ }^{1}$, riassunsi poi gli studi fatti, mostrando come il Bicaram consenta, tra l'altro, la massima utilizzazione delle reazioni induttive del suolo, opportunamente energizzato a c.a., a differenza delle apparecchiature molto meno sensibili (com'è anche subito intuibile) australiane e svedesi. Nel riassunto ricordo ruesto motivo fondamentale-strumentale. Schemi e foto illustrano tale complesso strumentale con un "modello" di compensatcre a c.a. pel confronto rapido, in grandezza e fase, dei campi m. alternativi di uguale frequenza, dovuti a suoli interessati da c.a. impressa induttivamente o galvanicamente.

Ho voluto completare, sia pure a distanza di tempo, (avendo in questo intervallo elaborato nuovi indirizzi d'indagine e.m. per cui lo stesso impiego del Bicaram ne $\dot{e}$ enormemente avvantaggiato) una serie d'esperienze in scala ridotla $\left({ }^{2}\right)$.

Devo a questo punto ricordare che $i$ nuovi indirizzi di rilevamento c.m., da me introdotti, c che hanno rimosso i massimi impedi- 
menti applicativi, (da cui la decadenza dell'impiego dei $\mathrm{m}$. induttivi, quasi subito dopo il loro avvento), tendono ad eliminare, nelle misure, l'addendo più ingombrante, ai fini presenti, del c.e.m. totale terrestre, e cioè il c.m. primario dell'emittore $H_{1}$, preponderante e che maschera le eventuali anomalie e.m. del suolo.

Migliorare il rapporto $H_{1} / H_{2}$, (dove $H$, è il c.m. secondario relativo ad es. ad un suolo anomalo con forte predominanza in generale di $H_{1}$ su $\left.H_{2}\right)$, non si può certo agendo sul generatore col rinforzare $H_{1},\left(H_{1} / H_{2}\right.$ rimane sostanzialmente costante), né agendo sulla sensibilità del ricettore (Bicaram), il cui aumento di sensibilità vale orviamente sia per il c.m. primario che per il c.m. secondario. Non si otterrebbe con ciò un risultato migliore agli effetti delle possibilità di svelare la presenza di conduttori specie deboli o profondamente situati.

Da qui la necessità di attenuare o eliminare l'azione del c.m. primario, finalità che si può raggiungere in più modi, in particolare con un recente metodo, definibile delle " zone d'ombra elettromagnetiche ", ottenuto con dispositivo che può dare tutt'intorno nello spazio circostante la somma: c.m.p. + c.m.s., ad eccezione che nella zona di misura ove trovasi il ricevitore (v. bibliografia) $\left(^{2}\right.$ ).

2. Ratiometer Turam, Bicaram. - La descrizione di un primo misuratore di rapporto di campi e. magnetici (c.e.m.) alternati di un suolo energizzato a c.a., in punti prossimi d'osservazione (" Ratiometer "), si trova già in Broughton Edge-Laby, I.G.E.S., Cambridge 1931. Commentando il "Ratiometer " gli autori inglesi osservano: il metodo rassomiglia sotto alcuni aspetti al sistema dei 2 telai equilibrati dovuto a Sundberg-Lundberg nel quali i 2 telai vengolo collegati in opposizione (Turam).

Per la descrizione del "Bicaram ", per il significato rigoroso dei simboli cbe qui appaiono, rimando ai miei lavori $\left({ }^{1}\right)$, ma perché si possa seguire facilmente quest'esposto è necessario che mi soffermi un poco sul compensatore a resistenze: 2 telai o bobine uguali, I, II, ad es. orizzontali, complanari, a breve distanza reciproca, in opposizione, con amplificatore e telefono nel circuito di equilibramento.

La bobina I è chiusa, in corto circuito, su una resistenza ohmica costante $R$; un cursore mobile "scorrevole su $\mathrm{R}$ ) permette di ricavare frazioni qualsiasi della f.e.m. indotta dal c.m. del suolo nella bobina I, con dispositivo potenziometrico. I diversi valori di $R,\left(R_{1}\right.$,

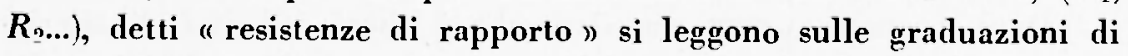
un cosidetto quadrante di rapporto, in unità convenzionali, o con 
preventiva taratura, in effettive resistenze in ohm corrispondenti alle divisioni del quadrante.

La bobina II è chiusa sopra una resistenza ohmica fissa $T_{0}$ e su una resistenza variabile $S$, "resistenza di fase ": i valori $S_{0}, S_{1}, S_{2}, \ldots$ di questa si desumono pure dalle divisioni di un quadrante di fase.

I due circuiti I, II, (qui come nel Bicaram), sono collegati attraverso i primari di due trasformatori d'isolamento allo scopo d'eliminare le correnti vaganti dovute a difetti d'isolamento o ad effetti capacitivi: accorgimento necessario in tutti gli strumenti del genere.

Rapidamente ricordo che nel Bicaram mentre la bobina $I$ è in serie con una resistenza e un condensatore fissi, la bobina II è in serie con resistenze e condensatori variabili.

In “Messungen mit dem Schleifdraht-Wechselstrom-Kompensator», precisamente nel paragrafo: " Fehler durch Isolations und Kapazitatsströme ", W. Geyger $\left({ }^{3}\right)$ così s'esprime: "facendo misure in c.a. possono notoriamente prodursi errori considerevoli per effetto di correnti d'isolamento e di capacità. Questi errori sono sopprimibili avendo cura che nel complesso delle misure e del dispositivo le parti in gioco non possiedano differenze di potenziale troppo grandi rispetto al mezzo circostante. Un modo adatto a ciò, in certi casi, è l'inserzione di trasformatori con avvolgimenti molto bene isolati tra loro e collegati con una terra messa in posizione opportuna. Il circuito di compensazione propriamente detto, incluso quello del galvanometro a vibrazione, (ovvero del telefono), deve isolarsi per mezzo di un trasformatore d'isolamento, che ha lo scopo d'impedire che tra la sorgente di corrente e il circuito di compensazione compaiano correnti d'isolamento o capacitive ".

I secondari vengono chiusi attraverso un amplificatore, su una cuffia telefonica, oppure su un galvanometro a vibrazione (es. Schrering Schmidt) o a risonanza, che, com'è noto, rimane a zero se percorso da correnti di frequenza maggiori a quelle su cui è sintonizzato.

È opportuno disporre di un dispositivo di variazione che permette d'innalzare o di abbassare di tono le frequenze del galvanometro in modo facile e opportuno. In parecchi casi si collega il galvanometro (o il telefono), messo a terra, al circuito di compensazione attraverso un trasformatorino isolato elettrostaticamente.

Le bobine I, II, complanari, convenientemente distanziate, immerse in $2 \mathrm{c} . \mathrm{m}_{\text {. del suolo }} H_{1}, H_{3}$ di ugual frequenza, (diversi in grandezza e fase), saranno sedi di due diverse f.e.m. indotte. Agendo opportunamente sulle resistenze variabili $R, S$, si “compensa " la corrente 
nel circuito I con quella che circola in II, ciò che viene rivelato ad es. dal silenzio nel telefono: allora le f.e.m. si riterranno uguali.

Dalle letture dei 2 quadranti, (e dalle costanti strumentali), ¿̀ immediato ricavare il rapporto tra le ampiezze dei campi $H_{1}$ e $H_{2}$ e la loro differenza di fase. La corrente $i_{1}$ in $I$ è uguale al rapporto: $e_{1} / Z_{1}$, (fra la f.e.m. indotta " $e_{1}$ ) e l'impedenza $Z_{1}$ ), a sua volta: $e_{1}=-d \omega_{1} / d t$, dove il flusso concatenato: $\omega_{1}=h_{1} S(S=$ area della bobina, $h_{1}=H_{1}$ sen ( $\left.(1)-q_{1}\right)$, intensità del campo $H_{1}$ all'istante $t$ ). Risulta che, a meno d'una costante “ $m$ ", “ $\left.e_{1}\right)$ è proporzionale a $H_{1}$ infatti: $e_{1}=m H_{1}$. Detta $R_{0}$ la resistenza complessiva del circuito $I_{\text {, }}$ (costante perché somma di quella della bobina con tutta la resistenza di rapporto), $L$ il coefficiente di autoinduzione, la reattanza della boljina sarà : $X=2 \pi f L$, l'impendenza $Z_{1}=\left(X^{2}+R_{0}\right)^{1 / 2}$, perciò la corrente $i_{1}$ nella bobina $I$ (posta nel campo $\left.H_{1}\right): i_{1}=m H_{1} /\left(X^{2}+R_{0}^{2}\right)^{2} / 2$. La caduta di tensione: $V_{1}$ tra gli estremi della resistenza olımica $R_{1}$ percorsa dalla c. $i_{1}$ sarà : $V_{1}=R_{1} i_{1}$.

Nel circuito II, quando si assegna alla resistenza di fase un valore $S=S_{0}$ (all'incirca a metà di $S$ ), la resistenza ohmica complessiva in serie con la bobina II è uguale a $R_{0}$. Per ogni altro valore della $S, S_{1}$, tale resistenza subisce un incremento, ciò̀ diventa: $R_{0}+S_{1}-S_{0}$.

L'impedenza $Z_{2}$ del circuito II essendo: $\left[X^{2}+\left(R_{0}+S_{1}-S_{0}\right)^{2}\right]^{1 / 2}$ la corrente ̀̀ : $i_{2}=e_{2} / Z_{2} ;$ a sua volta " $e_{2}$ " è proporzionale, con lo stesso fattore di proporzionalità, precedente al campo $H_{2}$, quindi: $i_{\underline{2}}=m H_{2} / Z_{\text {o. }}$.

La caduta di tensione $V_{9}$ agli estremi della resistenza $T_{0 \text {, percorsa }}$ dalla corrente $i_{2}$ è: $V_{2}=\tilde{i}_{0} i_{2}$.

A compensazione ottenuta sarà $V_{1}=V_{2}$, da cui :

$$
\frac{H_{1}}{\bar{H}_{2}}=\frac{\underline{T}_{0}}{r_{1}} \cdot\left(X^{2}+R_{0}^{2}\right)^{1 / 2} \cdot\left[X^{2}+\left(R_{0}-S_{0}+S_{1}\right)^{2}\right]^{1 /,}
$$

Gli sfasamenti fra tensione e corrente nei circuiti I e II rispettivamente saranno: $\varphi_{0}=\operatorname{artn} \mathrm{X} / R_{0}, \varphi_{1}=\operatorname{artn} X /\left(R_{0}-S_{0}+S_{1}\right)$, e lo sfasamento relativo delle 2 correnti, (e quindi dei 2 campi $H_{1}, H_{2}$ ), $\alpha=r_{0}-r_{1}$.

Le espressioni che danno $H_{1} / H_{2}$, e $a$ risolvono il problema in funzione delle costanti strumentali e di $R$ e $S$ variabili, conforme a quanto già dedussi come caso particolare nella mia teoria generale $\left({ }^{1}\right.$ ) del 1942.

3. Prime esperienze in scala ridotta con il compensatore a ca. 
Bicaram. - Le esperienze sono state condotte secondo i segucnti criteri generali :

a) produrre un campo magnetico primario alternativo, (di frequenza acustica $500 \mathrm{H}_{\mathrm{z}}$ ), mediante un "cappio energizzante ", o un emittore galvanico-induttivo, studiare $i$ vari emittori in relazione alall'impiego del compensatore;

b) riconoscere e valutare mediante il Bicaram il campo magnetico (c.m.) risultante in superficie del suolo dalla somma dei campi m. agenti, tra cui quelli provocati dalle correnti indotte (vorticose) nella massa conduttrice di un modello;

c) riassumere e rappresentare, mediante tabelle, grafici, diagrammi, i risultati delle misure e ricavarne utili norme per la prospezione effettiva sul terreno.

Nel mio studio "Sulle prospezioni e.m. di alcune tipiche giaciture di solfuri misti in Sardegna " del $1948\left({ }^{1}\right)$ ho discusso in particolare la modellometria elettromagnetica e un metodo di prospezione che potreblue derivarne (Elmod), particolarmente idoneo per i giacimenti sardi o del tipo sardo.

La scelta del terreno per le esperienze non dovreblue mai essere subordinata a ragioni di convenienze pratiche, e cioè prossimità ad es. degli Istituli attrezzati, con prese di energia elettrica dalle linee industriali, vasche di prova fisse, ecc., e questo perché, specie in centri urbani, la circolazione delle "correnti vaganti " nel suolo, è spesso abbastanza intensa per compromettere o falsare gli effetti da misurare.

In un primo tempo mi sono avvalso come generatore di un alternatore a frequenza $500 H_{z}, n=20.000$ giri al primo, tensione ai morsetti: 200 volt, potenza $1 \mathrm{KvA}$ circa, eccitazione mediante batteria di accumulatori (4 Volt-5 Amp.), con motore trifase da 160 Volt, $f=42 H_{z}$, potenza $1 \mathrm{KvA}$ circa, (e questo in mancanza d'un gruppo a motore a scoppio-alternatore).

Il doversi collegare ad una rete di distribuzione di energia ad uso industriale, (per alimentare l'alternatore e l'amplificatore), si rilevò, nel corso delle misure, un inconveniente abbastanza serio.

Per mantenere costante quanto più possibile la frequenza della corrente impiegata, e poter far fronte sufficientemente alle inevitabili oscillazioni della frequenza industriale usata nel motore, $i$ due organi motore e alternatore furono accoppiati mediante cinghia scorrevole su 2 pulegge tronconiche. Una guidacinghia azionata da una vite a piccolo passo permetteva di apportare alla velocità angolare dell'alternatore variazioni anche piccole e ben regolabili. Un tachimetro conta- 
giri solidamente montato sull'albero dell'alternatore permetteva di seguire e correggere agevolmente e prontamente gli scarti della frequen. za: nel corso delle misure questi scarti non risultarono mai superiori all' $1 \%$.

Poiché l'equilibrio nel compensatore si giudica in genere e con sufficiente approssimazione in base ai minimi di suono nel telefono, si eliminarono fin da principio le armoniche di ordine superiore, interponendo fra il circuito di utilizzazione e il generatore un filtro " passa-basso ". La potenza disponibile ai morsetti di uscita del filtro, notevolmente inferiore a quella erogata dall'alternatore, risultò sempre

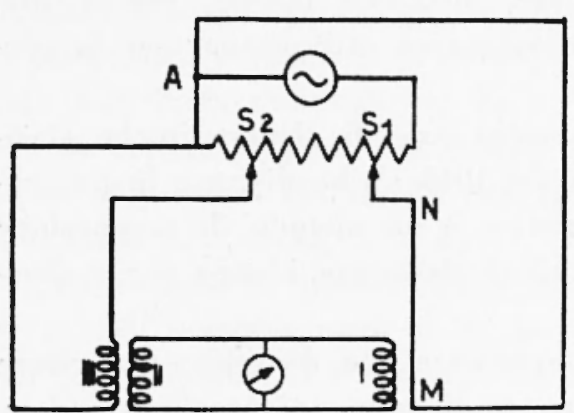

Fig. 1

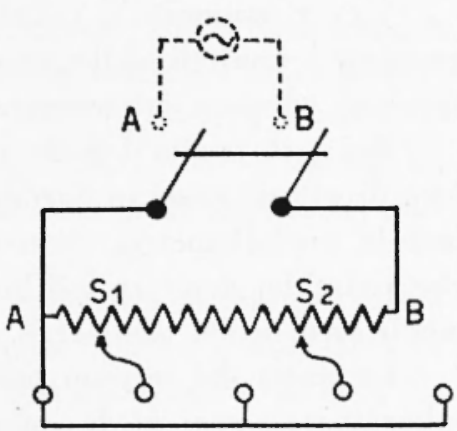

Fig. 2

però largamente sufficiente per alimentare $i$ circuiti secondo $i$ vari schemi adottati e le esigenze delle misure.

Circuiti di misura. - Nell'c Impiego del compensatore a c.a. Bicaram nelle misure a scala ridotta" (1) mostrai come sia opportuno un adattamento strumentale, ma non ben inteso come è previsto sul terreno: basta ad es. manovrare una sola bobina esploratrice I, mantenendo fissa e distanziata la bobina compensatrice II (v. Fig. 1).

Intensità e fase della corrente nella bobina $I$ vengono riferite a quelle della corrente circolante nella bohina II, e ciò ottenni nel modo più semplice mediante l'inserimento di una bobina III (inducente) rigidamente collegata alla II (cosi da assicurare una mutua induzione costante), e alimentata dal circuito potenziometrico che chiude il generatore.

Un amplissimo anello di filo di rame rivestito (mm. 1,5) costituiva il circuito del cavo induttore $A M N S_{1}$; con un tratto sui $30 \mathrm{~m}$, $M N$ sopraelevato di $1 \mathrm{~m}$ dal suolo, rettilineo e orizzontale.

La regolazione della corrente in questo cavo $e$ in quello induttore della bobina III avveniva mediante il dispositivo potenziometrico indicato in Fig. 2, realizzato montando su un pannello di bachelite un 
interruttore generale della linea dell'alternatore, e tra i morsetti $A$ e $B$ una resistenza variabile costituita da un gruppo di bobine di varia resistenza, (ad avvolgimento antinduttivo), e da un reostato a cursore per complessivi $1100 \mathrm{Ohm}$; l'insieme dei circuiti e del reostato venne isolato dapprima dal suolo, con isolatori in "pyrex", appoggiando su carrucole di porcellana sia il gruppo generatore, che il resto.

Non appena s'inviò corrente però fu impossibile effettuare qualsiasi esatta misura, nonostante che il provacircuiti desse indicazioni di

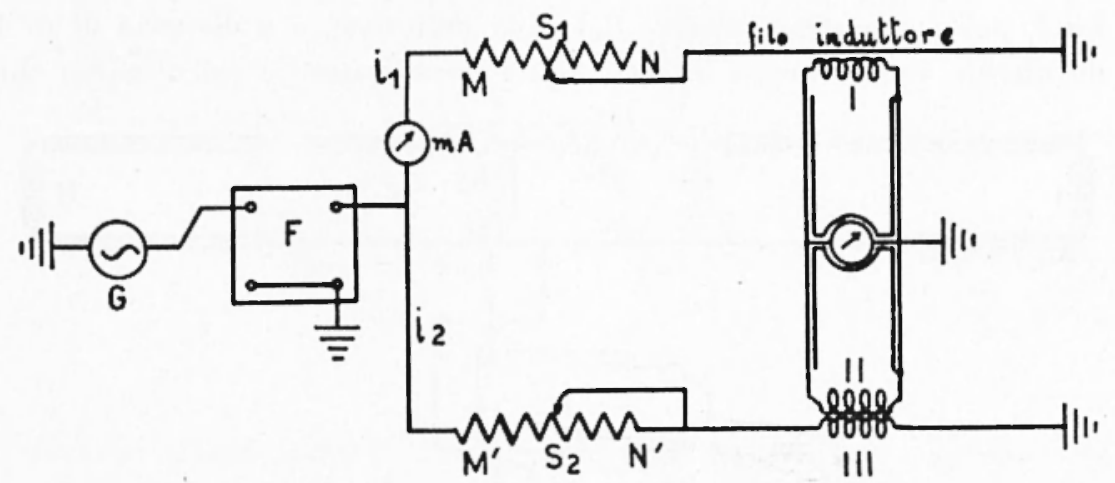

Fig. 3

resistenza verso terra dell'ordine di $20 \mathrm{Mohm}$ e anche più. Si pensò ad effetti di correnti capacitive e ad inavvertiti difetti d'isolamento intercircuitali tali da prevalere e da mascherare ogni e qualsiasi altro disturbo. Lo stesso pannello di bachelite con le resistenze poteva diventare una sorgente sospetta e malsicura di f.e.m. estranee. Si preferì allora adottare il criterio di una razionale messa a terra dei vari organi e dei vari circuiti, nonché la schermatura degli apparecchi mediante un ancllo di guardia messo a sua volta a terra: non si era voluto fin da principio seguire questa via per timore che tali collegamenti al suolo potessero influire sulle misure: (v. Fig. 3), il che infatti avverrebbe senza un preliminare esame delle modalità più opportune delle stesse messe a terra.

Un polo dunque del generatore $G$ al suolo; 2 circuiti derivati dall'altro polo pure a terra attraverso le 2 resistenze variabili antinduttive $M N$ e $M^{\prime} N^{\prime}$. Il cursore $S_{1}$ regola l'intensità della corrente $i_{1}$, nel filo induttore della bobina I (esploratrice); il cursore $S_{2}$ quella della corrente $i_{2}$ nel circuito della bobina III accoppiata (in aria) alla bobina II (compensatrice).

Quanto al compensatore, la cassetla racchiudente $i$ vari elementi di misura (condensatori fissi e variabili, resistenze, ecc.) venne fode- 
rata con $A l$ con morsetto di presa per terre, cosi le 2 bobine, l'armatura esterna del cavo schemato di collegamento bolsine-compensatore, l'amplificatore schermato e il supporto della cuffa telefonica, il tutto al suolo. Lo schema del compensatore e quello dell'amplificatore appaiono in queste condizioni, in Fig. 4.

Dopo questi esami e disposizioni strumentali s'iniziò a studiare il campo e.m. prodotto dal cavo percorso dalla corrente $i_{1}$ nella sola bobina $I$, (separata per il momento dal resto del compensatore). La $I$, posta alla stessa altezza dal cavo induttore, e a distanza di m 1 da questo, veniva messa in serie con l'amplificatore e col telefono che

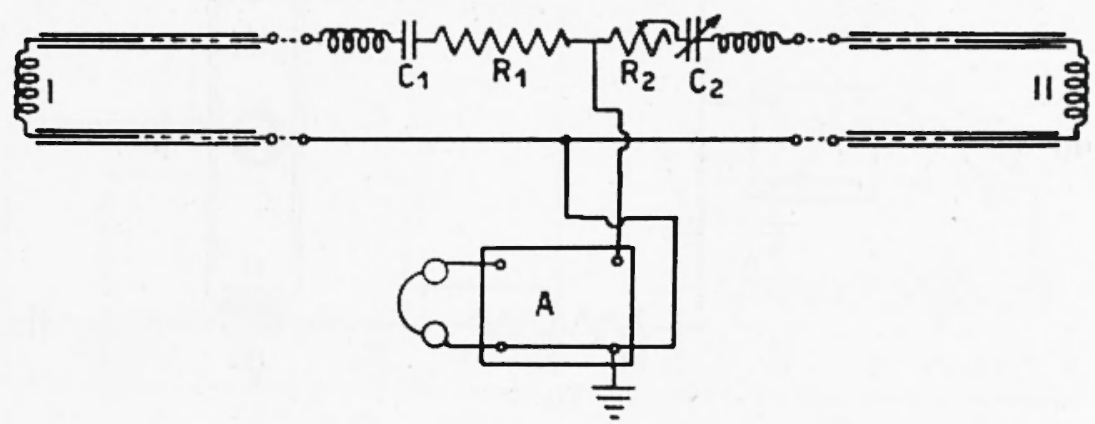

Fig. 4

accusava un suono fortissimo, tanto che per agevolare la ricerca preliminare dei minimi riuscì molto vantaggioso sostituire alla cuffia telefonica un misuratore di potenza d'uscita ad alta impedenza (40000 Ohm), con 6 diverse sensibilità, da usare come indicatore di zero. Finalmente si ottenne un'indicazione nitida e non influenzata dalla vicinanza dell'osservatore alla bobina, o alle manopole, o ai comandi dell'apparecchio. Ruotata la bobina di $90^{\circ}$, la deviazione nello strumento scompariva quasi completamente. La cuffia, nuovamente sostituita all'indicatore, confermò che si aveva una fortissima attenuazione, se non il silenzio perfetto. Si passò poi a compensare le correnti indotte nelle bobine I e II col mantenere fissa la posizione della I (in un campo di 10 « Oe.) e col variare l'intensità della corrente inducente $i_{\text {s nel reostato }} M^{\prime} N^{\prime}$; dapprima le bolbine erano chiuse in opposizione attraverso l'amplificatore e il misuratore secondo lo schema di Fig. 5, in seguito si usò il telefono, ottenendo un equilibrio approssimato dopo qualche tentativo di manovra.

A questo punto le 2 bobine venivano collegate al compensatore nel quale si era predisposta la lettura: $\varphi=0^{\circ}, K=1=\frac{\left|Z_{1}\right|}{\left|Z_{2}\right|} \quad$ (giac- 
ché era da presumere che le correnti nelle bobine risultassero uguali in grandezza e concordi di fase). Senonché perdurando le misure non conformi alle previsioni, nonostante tutte le precauzioni prese per eliminare l'influenze previste, proprie del complesso strumentale, (il silenzio s'aveva con sensibili modificazioni nelle costanti strumentali, come se la risonanza per ciascuna bobina non si avesse già per $C=67,55 \mathrm{~m} \mu \mathrm{F}$, secondo i risultati del calcolo, ma per valori alquanto
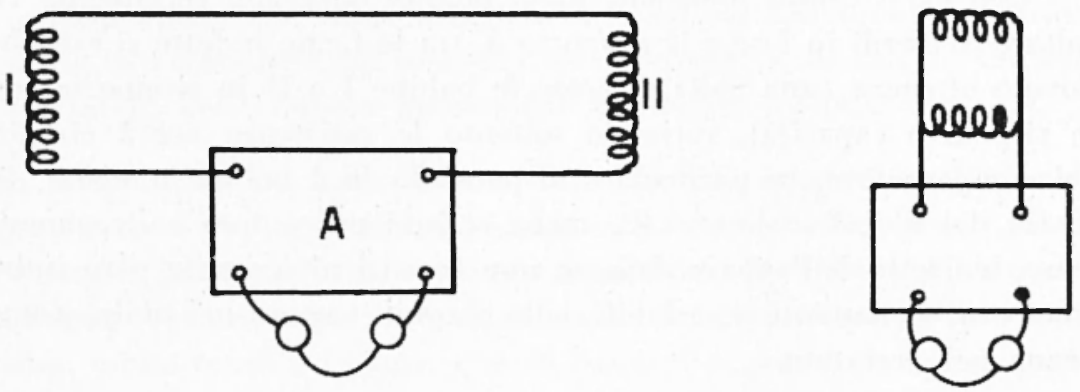

Fig. 5.

diversi; scambiando i collegamenti delle bobine col compensatore $i$ valori di misura risultavano differenti, confermando che le correnti nelle boline non concordavano in fase), si cercarono altre cause, perturbanti, quali ad es. il vincolo delle misure in località di sperimentazione con linee elettriche per uso industriale, ed altre.

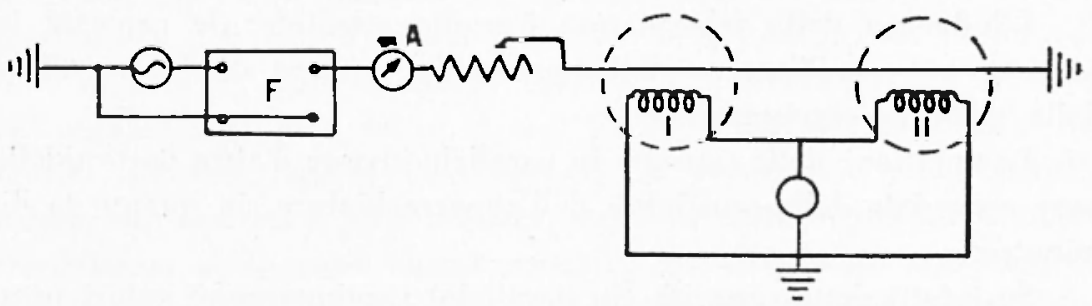

Fig. 6

Si volle insistere ancora nelle prove lasciando fissa la corrente $i$. e la bobina II (compensatrice), e allontanando la I dal filo in modo da provocare una diminuzione (in rapporto noto) della corrente nella I stessa, ma risultarono alterati in maniera affatto arbitraria e imprevedilile i rapporti e le fasi. Poiché l'accoppiamento induttivo tra le bobine II e III poteva sembrare troppo stretto in confronto a quello tra il filo $i_{1}$ e la bobina I (Fig. 3), rendendo malsicuro e disagevole l'esatta misura della corrente $i_{\text {, }}$, si diminuì il numero delle spire co- 
stituenti la III, sino a ridurle ad una sola, ma i miglioramenti furono inapprezzal,ili.

Un'ulteriore semplificazione del circuito induttore, per eliminare altre cause di "dispersione » si apportò immergendo la bobina compensatrice II nello stesso campo inducente della bobina $I$, sostituendo nello schema di Fig. 3 quello di Fig. 6.

Con ciò i campi inducenti nelle bobine dovevano certamente risultare concordi in fase e il rapporto $K$ tra le f.e.m. indotte si sarebbe dovuto ottenere (una volta disposte le bolsine I e II in risonanza con le rispettive capacità), variando soltanto le resistenze nei 2 circuiti del compensatore, in particolare disponendo le 2 bobine a ugual distanza dal filo, $K=1, \uparrow=0^{\circ}$, anche scambiando i loro collegamenti con i morsetti dell'apparecchio, se non fossero intervenute però induzioni e.m. sconosciute e variabili delle correnti vaganti nel suolo,. come venne poi accertato.

Capacità parassite introdotte. - Altro problema è l'influenza della schermatura sulle capacità del compensatore, ossia determinare le condizioni di risonanza del sistema formato da ciascuna bobina, dai condensatori in serie con esse, e in più dal cavo schermato di collegamento.

La schermatura del cavo, equivalendo ad un consensatore in parallelo con quelli montati nell'interno dello strumento non permette la risonanza pel valore prima calcolato, ma per $C=63 \mathrm{~m} \| F$ circa.

L'influenza della schermatura è molto sensibile: le capacitì in serie $C_{1}$ e $C_{2}$ nell'interno dell'apparecchio vennero diminuite allora dalla quantità corrispondente.

La questione delle capacità in parallelo investe d'altra parte quella pure essenziale della sensibilità dell'apparecchiatura, in quanto la diminuisce.

Se infatti dette capacità (in parallelo) raggiungessero valori prossimi alla risonanza dei circuiti, (ciò accade tanto più facilmente quanto maggiore è la frequenza, per $f=500 \mathrm{~Hz}, L=1 \mathrm{H}, C=10^{-i} \mathbf{F}$ ), la compensazione si otterrebbe con difficoltà, con enormi contrasti delle resistenze ohmiche di manovra. Per quanto qui si tratta di capacità in parallelo " equivalenti ", con funzioni di semplici attenuazioni delle capacità in serie, tuttavia la taratura dei circuiti, (soprattutto per ciò che riguarda le capacità occorrenti per la sintonia e per la compensazione di fase), non può esser fatta soltanto in base ai dati costruttivi delle bolnine impiegate.

La capacità propria dei cavi colleganti ciascuna bobina all'appa- 
recchio richiede una compensazione particolare per la bobina a impedenza fissa, e per l'altra bobina una verifica con l'aiuto dell'oscillografo a raggi catodici. E ciò sempre dopo la messa a terra degli schermi delle bobine, (lamierino sottile di ottone con fenditure radiali), insieme con l'armatura dei cavi schermati, per attenuare, prima di estinguerli, sia pure con l'uso delle capacità " equivalenti ", gli effetti capacitivi parassiti.

Disponendo le bolsine a eguali distanze dal filo induttore (schema Fig. 6) l'equilibrio del compensatore non veniva alterato invertendo gli attacchi delle bobine ai morsetti del condensatore.

Riconoscimento della natura delle correnti vaganti. - Per spiegare i residui risultati anomali, nonostante i correttivi introdotti, bastò fermare il generatore di corrente e constatare che nelle bobine continuavano a circolare correnti d'intensità rilevante, capaci di produrre campi molto estesi e intensi. Queste correnti si potevano d'altra parte elidere (per opposizione), se stazionarie, dando alle bobine orientazioni qualsiasi, purché parallele tra loro e a distanza non grande ( 4 o $5 \mathrm{~m}$ ).

Per riconoscerne la natura si adottò un circuito semplicissimo di esplorazione, costituito da una delle boline dell'apparecchio, chiusa sulla cuffia telefonica attraverso l'amplificatore, mantenendo inattivo il greneratore a $500 \mathrm{ffz}$. Risultò che le c.a. molto intense, circolanti nel suolo, non avevano la frequenza solo di $42 \mathrm{~Hz}$ (come si poteva prevedere per l'allacciamento del motore), ma anche molto più elevate, prossima a $250 \mathrm{~Hz}$. Variando l'aliezza della bobina dal suolo, Ida $\mathrm{m} \quad 0,50$ a $\mathrm{m} 3$, non si elbero variazioni apprezzabili nell'intensità del segrnale telefonico. Oltre al crepitio e al ronzio caratteristici dei motori elettrici (specie l'avviamento), si avvertirono "battimenti" fortemente marcati e prossimi alla frequenza 250 . Segnali delle radiotrasmissioni risultarono altresì sensibili, ma eliminabili con condensatori appropriati. Ruotando per tentativi la bobina, si riuscì per una certa orientazione, ad ottenere silenzio quasi completo, con campo prevalente polarizzato ellitticamente in un piano verticale, campo non stazionario, perché ad intervalli di tempo molto irregolari (di primi o di secondi) ne variavano i caratteri, rendendo così incerta o impos. sibile una precisa determinazione.

Di questi caratteri alcuni si potevano interpretare senza difficoltà, soprattutto quelli relativi al "campo di fondo" di $42 \mathrm{~Hz}$, di altri l'interpretazione riusciva più complicata, specie riguardo l'esistenza di campi ad alta frequenza e di battimenti nitidamente rilevati. 
L'insieme delle correnti circolanti nel sottosuolo di una cittá specie industriale $\dot{e}$, in genere, a diverse frequenze, estremamente complesso e non stazionario. Il c.m. complessivo relativo risulta d'intensita tale da falsare o mascherare le osservazioni del campo (precalcolato) del nostro induttore, come pure d'induttori analoghi.

Da qui l'opportunità per l'esecuzione delle misure di cercare zone meno perturbate, con ricognizioni preliminari sia pure con una sola bobina, amplificatore, cuffia telefonica, oltre relativo cavo schermato di collegamento e quello per gli allacciamenti dell'amplificatore.

In una delle tante prove preliminari, lontano da centri urbani, (tenendo conto della esperienza acquisita), l'amplificatore venne allac-

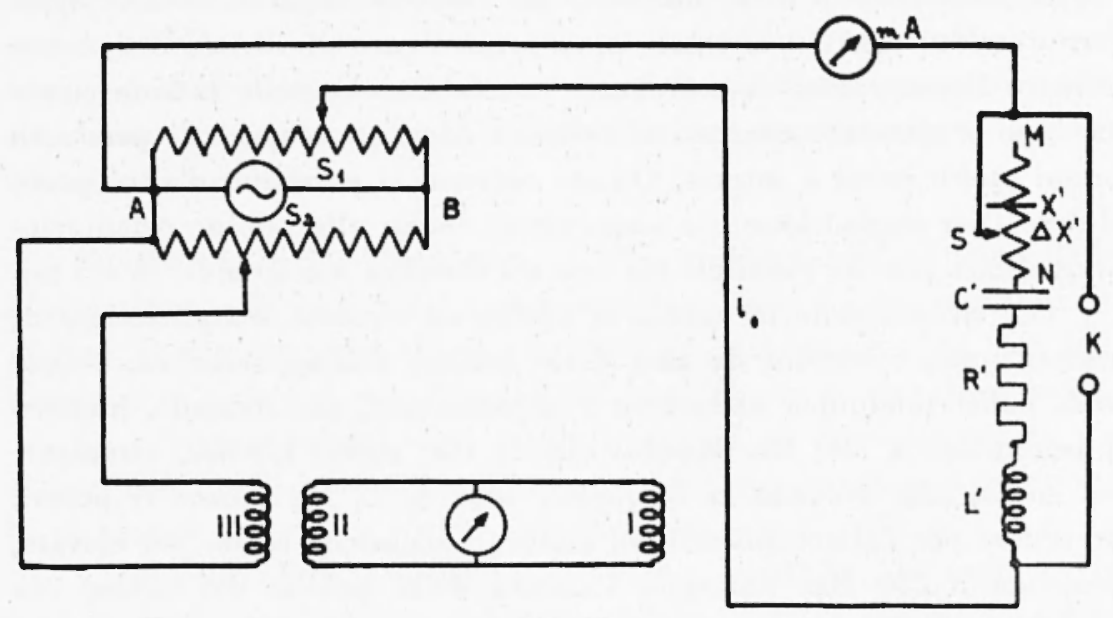

Fig. 7

ciato ad una rete luce a $42 \mathrm{~Hz}$ e 130 Volt. La bobina poliassiale fu collocata in diversi punti della zona, e in ciascuna posizione ruotata opportunamente fino ad ottenere il silenzio nel telefono: il suono del telefono rivelò che esisteva dapertutto un campo di fondo a $42 \mathrm{~Hz}$, ma non un campo a frequenza più elevata. Ruotando la bobina di $360^{\circ}$ intorno ad un asse orizzontale (normale al piano di silenzio) si trovarono 2 massimi e 2 minimi assai deboli, con l'asse minore (orizzontale) dell'ellisse di polarizzazione molto piccolo rispetto a quello verticale. Altri rumori a bassa frequenza vennero pure blandemente avvertiti (crepitii, avviamento di motori, forse per macchine utensili, di sollevamento d'acqua ecc.).

Queste esperienze comunque mi dettero il modo, non raquiungendo subito i fini propostimi, (sia pure per cause estranee), di studiare e 
introdurre nel Bicaram (capacità equivalenti ) per eliminare o attenuare quelle parassite distribuite, (notevole e indispensabile accorgimento a vantaggio della sicurezza strumentale), prima delle operazioni di “ messa a terra ", non implicanti perciò, per la tenuità degli effetti, ulteriori problemi.

4. Adattamenti del Bicaram nelle misure in scala ridotta. - Lo schema che risultò più idoneo, per le ragioni esposte, ai fini qui preposti, è il seguente (Fig. 7):

Nel circuito derivato tra il morsetto $A$ del generatore e il cursore $S_{1}$, si dispose in serie col cavo induttore una bobina di rame, campione di induttanza fissa: $L^{\prime}=1$ Henry, la resistenza ohmica fissa $R^{\prime}=160 \mathrm{Ohm}$, un condensatore variabile $C^{\prime}$, una resistenza ohmica $X^{\prime}$, una chiave di corto circuito $K$, (derivata agli estremi di questa serie) simbolo da non confondere con il $K$ dei rapporti.

E chiaro che chiudendo $K$ tutta la serie precedente viene ad essere esclusa dal circuito, e si ritorna allo schema consueto, ma tenendo aperta $K$, si possono attribuire ad $L^{\prime}, C^{\prime}$, ed $X^{\prime}$ tali valori che l'induttanza del circuito assuma il valore: $M^{\prime}=\omega L^{\prime}-\left({ }_{10} C^{\prime}\right)^{-1}$, provocando in esso uno sfasamento $\varphi$ fra f.e.m. $e$ corrente, dato da: $t n \varphi=M^{\prime} /\left(R^{\prime}+X^{\prime}\right)$, (si trascura la resistenza propria del filo induttore). L'induttanza $M^{\prime}$ dunque può essere variata a piacere e precalcolata (almeno con approssimazione), mentre variando la posizione del cursore $S_{2}$ del reostato che chiude i morsetti del generatore, si può variare l'intensità della corrente nel cavo.

Dunque il dispositivo si presta ad apprezzare il comportamento e la sensibilità del compensatore per valutare il rapporto tra le f.e.m. indotte nelle bobine I e II e le relative differenze di fase.

Il valore di $C^{\prime}$ (frequenza di $500 \mathrm{~Hz}$ ), corrispondente alla risonanza, ̀̀ dato da: $\omega^{2} L^{\prime} C_{0}{ }^{\prime}=1$ ossia: $C_{0}{ }^{\prime}=101,3.10^{-9} \mathrm{~F}$ per cui risulta naturalmente: $\psi=0$; variando $X^{\prime}$ si potrà valutare la sensibilità dello strumento come misuratore di rapporti $K$ d'intensità. Attribuendo a $C^{\prime}$ valori minori di $C_{0}^{\prime}$, la resistenza del circuito è capacitativa e $q$ risulta negativo; ad es. ponendo $C^{\prime}=95.10^{-9} \mathrm{~F} .<C_{0}{ }^{\prime}$ risulta: $M^{\prime}=-210$ Ohm.

Dalla: $t n \varphi=M^{\prime} .\left(R^{\prime}+X^{\prime}\right)^{-1}$, conviene ricavare $\mathrm{i}$ valori $\mathrm{di} \boldsymbol{X}^{\prime}$ che corrispondono a valori opportuni di $\varphi: X^{\prime}=M^{\prime}[\operatorname{tn}(-\uparrow)]-^{1}-R^{\prime}$.

Attribuendo invece a $C^{\prime}$ valori maggiori di $C_{0}$, la reattanza è induttiva e $M^{\prime}$ risulta positiva: $\operatorname{così~per~} C^{\prime}=110$ in $u$ F., è: $M^{\prime}=248$ Olın, $X^{\prime}=248$ tn $\uparrow-160$, da cui la tabellina: 


\begin{tabular}{|c|c|c|c|}
\hline$\varphi<0^{\circ}$ & $X^{\prime}$ & $\left(p>0^{\circ}\right.$ & $X^{\prime}$ \\
\hline$-2^{\circ}$ & $5854 \Omega$ & $+2^{\circ}$ & $6942 \Omega$ \\
\hline$-3^{0}$ & 3847 ) & $+3^{\circ}$ & 4572 ) \\
\hline$-4^{\circ}$ & 2843 ) & $+4^{\circ}$ & 3395 ) \\
\hline$-5^{\circ}$ & $2241 》$ & $+5^{\circ}$ & $2674 川$ \\
\hline$-10^{\circ}$ & $1031)$ & $+10^{\circ}$ & $1246)$ \\
\hline$-15^{\circ}$ & $623,7)$ & $+15^{\circ}$ & 765 \\
\hline$-20^{\circ}$ & $417,0 \quad)$ & $+20^{\circ}$ & $521 》$ \\
\hline$-30^{\circ}$ & 203,7 ) & $+30^{\circ}$ & $269 "$ \\
\hline$-40^{\circ}$ & 190,3 ) & $+40^{\circ}$ & 135 » \\
\hline$-50^{\circ}$ & $16,2)$ & $+50^{\circ}$ & $48 \%$ \\
\hline$-61^{\circ}$ & $0,0 \quad)$ & $+57^{\circ}$ & $0 \%$ \\
\hline
\end{tabular}

Ordine di grandezza delle correnti da impiegare nei circuiti induttori. - Le misure di prospezione sul terreno col metodo del cappio induttore si eseguono abitualmente alimentando il cappio con 3-4 Amp., e date le dimensioni comunemente attribuite a questo cappio nelle ricerche, l'intensità del campo m. nella regione centrale del cappio risulta di 50-100 $\mu \mathrm{O}_{e}$. Infatti nel centro geometrico di un cappio induttore rettangolare di lati: $a$ e $b$, si ottiene per il c.m.:

$$
H_{0}=\frac{4}{5} I a^{-1}\left[1+b^{2} a^{-2}\right]^{1 /,} ; \lim _{h \rightarrow \infty} \check{r}_{:}=\frac{4}{5} I \cdot a^{-1}
$$

dove $H_{\circ}$ si valuta in Oersted; $a, b$, in cm, $I$ in Amp. Se $a=400 \mathrm{~m}$, $b=1000 \mathrm{~m}, \quad b / a=\frac{5}{2}$. il radicale: $\left[1+b^{-} a^{-2}\right]^{1 / 2}=1,08$, perciò $H_{0}=2,15 \cdot 10^{-5} . I$, e per $I=3$ Amp., $H_{0}=65 \cdot 10^{-6}$ Oersted.

Adottando per le lunghezze la scala 1:500 (dimensioni del cappio in miniatura $\mathrm{cm}^{2}(5 \times 2)$, basta che la corrente nel filo si riduca a $3 / 500=0,006$ Amp. Si deve perciò prevedere una regolazione della corrente erogata dal generatore a pochi $m A$ : è preferibile per questo un dispositivo potenziometrico ad alta resistenza, che comprenda un amperometro in serie col generatore e con una resistenza antinduttiva.

Il generatore e il reostato si porranno lontani il più possibile dall'area dove si effettuano le misure: il filo del cappio sarà rivestito e bene isolato, di sezione sufficiente per assicurare la rigidià delle tesate.

Taratura del compensatore. - Si tende un cavo, (induttore orizzontale), sul suolo omogeneo prescelto per l'esecuzione delle misure, sollevato a $1 \mathrm{~m}$ circa da terra, e lo si alimenta tra il morsetto $A$ del 
generatore e il cursore $S_{1}$, dopo aver chiuso la chiave di c.c.K.

Si dispone la bobina I, (esploratrice), del compensatore orizzontale e complanare al cavo con la retta di graduazione azimutale $0^{\circ}-180^{\circ}$ normale al filo stesso, vedi Fig. 8.

Si pone dapprima $00^{\prime}=\mathrm{m} 1$, e si regola la corrente $i_{0}$ nel filo induttore, spostando il cursore $S_{1}$, fin quando il campo m. in $0^{\prime}$ assuma un valore prefissato unitario (ad es. $H_{0}=50$ oppure $100 \mu$ Oe.);

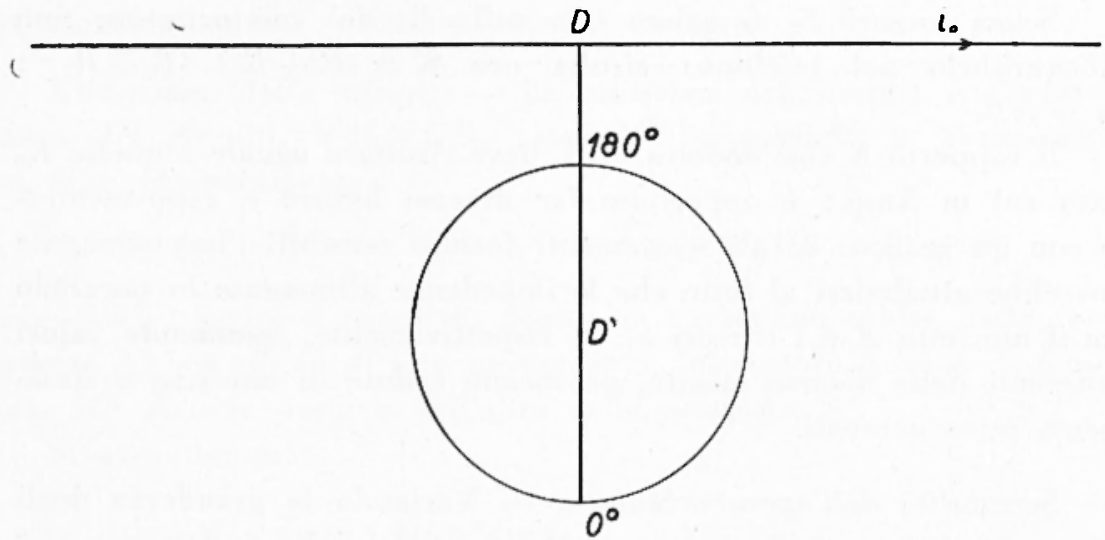

Fig. 8

la corrente $i_{0}$ letta sul milliametro, dovrà segnare rispettivamente, in valore efficace, 25 o $50 \mathrm{~mA}$.

Nel compensatore variabile si porta $C_{2}=C_{02}$ (valore di risonanza), si regola $R+R_{1}=R+R_{2}$, per cui risulta $K=1$, $=0$, ricordando [1] le semplici formole:

$$
\begin{gathered}
K=\left(R+R_{1}\right)\left[\left(R+R_{2}\right)^{2}+M_{2}^{2}\right]^{-1 / 2} \\
t_{\mathrm{n}} \varphi=M_{2} \cdot\left(R+R_{\mathrm{g}}\right)^{-1}, \quad M_{2}=(1) L-\left((1) C_{2}\right)^{-1}
\end{gathered}
$$

Allora si sposta il cursore $S_{2}$ fino ad ottenere silenzio nel telefono e fino a quando non saranno più toccati i cursori $S_{1}$ e $S_{2}$ lo strumento risulterà "tarato" in grandezza e fase rispetto al campo m. fondamentale $H_{0}$.

Ricerca di eventuali conduttori accidentali sul suolo scelto per le misure. - Allo stesso modo che si è reso indispensabile esaminare il comportamento del gruppo di misura senza eccitare il suolo con l'alternatore, prima d'iniziare le misure previste, si dovrà controllare, almeno in prossimità dell'induttore, che non si abbiano masse con- 
duttrici ed importanti invisibili da provocare un campo $\mathrm{m}$. secondario perturbatore: $h_{0}{ }^{\prime \prime}=H_{3}^{\prime \prime} \cos (\omega t-\alpha)$, dovuto alle correnti indotte in esse dal campo m. primario $H_{0}$, e a tale scopo si può procedere in diversi ben noti modi.

Accertata l'omogeneità del suolo si modifica l'intensità della $i_{0}$ variando la posizione del cursore $S_{1}$, e se ne legge il nuovo valore, $K_{0} \quad i_{0}$ sul milliametro $\mathrm{mA}$.

Senza toccare $S_{?}$ si agisce solo sulla $R_{2}$ del compensatore fino all'equilibrio nel telefono: risulta ora $K=\left(R+R_{1}\right) \cdot\left(R+R_{2}\right)^{-1}$; tn $\varphi=0$.

II rapporto $K$ cosi dedotto, $\neq 1$, deve risultare uguale a quello $K_{0}$ letto sul m Amp.; è opportuno far diverse letture e rappresentarle con un grafico; se gli scostamenti fossero sensibili l'inconveniente dovrebbe attribuirsi al fatto che le impedenze alimentate in parallelo tra il morsetto $A$ e i cursori $S_{1}, S_{2}$ rispettivamente, assumendo valori differenti nelle diverse misure, provocano cadute di tensione e sfasamenti pure notevoli.

Sensibilità dell'apparecchiatura. - Variando la grandezza degli elementi costituenti il circuito $A M N L S_{1}$ del cavo induttore, si ̀̀ in grado di variare, entro limiti abbastanza estesi, la grandezza e la fase di $i_{0}$ che lo percorre (senza modificare la grandezza e la fase della f.e.m. " $e_{0}$ ", applicata tra $A$ e $S_{1}$ ) e quindi anche del flusso concatenato con la bobina $I$ del compensatore.

Prefissato come prima un campo m. fondamentale $H_{0}$, si notrà determinare i valori da attribuire agli elementi del circuito induttore in modo tale che il nuovo flusso concatenato con $I$ stia in rapporto noto di grandezza e di fase con $H_{0}$. Il compensatore dovrà far ritrovare questi valori cosi prestabiliti e noti a priori, rendendo agevole lo studio della sensibilità strumentale nelle più diverse condizioni di misura.

Si procede in modo sostanzialmente simile a quello per la scelta di $H_{n}$ e la taratura dello strumento: si porrà $C^{\prime}=C_{o}^{\prime}$ la bobina orizzontale e complanare al filo induttore a distanza di $100 \mathrm{~cm}$; alla resistenza $M N$ si attribuirà un certo valore $X^{\prime}$ prescelto con criterio di opportunità, tra cui il tener conto dell'influenza della temperatura sulla resistenza della bobina $L^{\prime}$. A questo scopo si dispone in serie con ' $L$ ' una resistenza variabile a seconda le temperature, e tale che la somma di essa e della resistenza della bobina $L^{\prime}$ sia costante, ed uguale a $180 \mathrm{Ohm}\left(R^{\prime}=180 \mathrm{Ohm}, X^{\prime}=180 \mathrm{Ohm}\right.$, oppure $X^{\prime}=2.180$ 
Ohım a seconda i valori che si vorrà attribuire a $K$ ). Poi si sposterà il cursore $S_{1}$ fino a che la corrente $i_{0}$ letta sul $\mathrm{mA}$ raggiunga il valore voluto $\left(25\right.$ o $50 \mathrm{~mA}$, a seconda che si vuole che sia $H_{0}=50$ oppure 100 н Oc.l.

Si tara il compensatore su questo campo nel modo già visto; nel seguito tenendo fissi i cursori $S_{1}, S_{2}$ (nei limiti di approssimazione voluta) si potrà ritenere che le f.e.m. $e_{0}, e_{1}$, applicate tra $A S_{1}, A S_{0}$, si mantengano costanti e sempre concordi in fase.

Esecuzione delle misure. - La reattanza complessiva e l'impedenza del circuito $A M N L S_{1}$, saranno (trascurando la resistenza del filo) rispettivamente:

$$
M^{\prime}=(1) L^{\prime}-\left(\text { (n) } C^{\prime}\right)^{-1} \quad ; \quad Z^{\prime}=R^{\prime}+X^{\prime}+\Delta X^{\prime}+j M^{\prime} \quad ; \quad j^{\prime \prime}=-1
$$

dove $\Delta X^{\prime}$, positiva o nerativa, denota la variazione subita dalla resistenza $X^{\prime}$ per effetto di uno spostamento del cursore $S$ lungo il reostato $M N$ da una parte o dall'altra della posizione $X^{\prime}$.

Si arrà dunque:

$$
\begin{gathered}
Z^{\prime}=\left[\left(R^{\prime}+X^{\prime}+\Delta X^{\prime}\right)^{2}+M^{\prime 2}\right]^{\prime \prime} ; \quad i_{0}=e_{0} \cdot\left(R^{\prime}+X^{\prime}\right)^{-1} \quad ; \quad|i|=\frac{e_{0}}{\bar{L}^{\prime} \mid} \\
K=\frac{i}{i_{0}}=\frac{R^{\prime}+X^{\prime}}{Z^{\prime} \mid} ; \quad I_{\mathrm{n}}\left(p=M^{\prime} \cdot\left[R^{\prime}+X^{\prime}+\Delta X^{\prime}\right]^{-1}\right.
\end{gathered}
$$

Anche i campi magnetici dovuti alle correnti $i$ e $i_{0}$ staranno nel rapporto $K$ (tra le ampiezze) e (differenza delle fasi).

Orbene, lasciando invariata la posizione della bobina esploratrice $I$, il compensatore dovreblue misurare gli stessi valori $K$ e $\varphi$ suscritti.

Risolvendo rispetto a $X^{\prime}+\Delta X^{\prime}$ e a $M^{\prime}$ le 2 ultime equazioni che danno $K$ e $\varphi$, si ottiene facilmente:

$$
X^{\prime}+\Delta X^{\prime}=K^{-1}\left(R^{\prime}+X^{\prime}\right) \cos \varphi-R^{\prime} \quad ; \quad M^{\prime}=K^{-1}\left(K^{\prime}+X^{\prime}\right) \text { sen } \varphi
$$

formole clie permet tono di predeterminare quali valori si debbano attribuire a $X^{\prime}-\Delta X^{\prime}$ e a $C^{\prime}$ nel circuito del filo induttore, affinché il campo da esso prodotto stia nel rapporto $K$ (fra le ampiezze) e $\varphi$ (di differenza di fase) rispetto a $H_{0}$.

In queste esperienze $K$ e $\varphi$ si assegnarono ad arbitrio; p. es.: $K=0,4 ; 0,5 ; . .1 ; 1.1 ; 1,2 ; . .1,9 ; 2 \cdot \varphi= \pm 0^{\circ},-5^{\circ}, 10^{\circ}, . . \pm 45^{\circ} . .60^{\circ}$

Dai grafici già costruiti per l'uso del compensatore si ricavano $i$ valori di $R$ : e $C$.: che corrispondono a tali valori di $K$ e r. Qualora nella effettiva esecuzione delle misure, si ottenga l'equilibrio nel com- 
pensatore con valori $R_{2}^{\prime}, C_{2}^{\prime}$, sensibilmente diversi da quelli " previsti col calcolo ", sarebbero anebe diversi i valori $K^{\prime}$ e $\varphi^{\prime}$ forniti dallo strumento.

I rapporti: $\left(K-K^{\prime}\right) / \mathbf{K} ;\left(\psi-\psi^{\prime}\right) / \varphi$, danno un'idea dell'errore relativo inerente all'uso dello strumento nelle diverse regioni di misura.

Assumendo, come si ̀̀ detto, $R=180 \Omega, x=180 \Omega$, oppure: $R=180 \Omega, x=2.180 \Omega$ le espressioni precedenti (a parte gli apici) diventano, nel $1^{\circ}$ caso:

$$
X+\Delta X=180(2 \cos q / K-1) \quad, \quad M=360 \cdot \frac{2 \operatorname{sen} \psi}{K},
$$

formole adatte per piccoli valori di $K$, tra 0,4 e 1,2 ; oppure:

$$
X+\Delta X=180(3 \cos \Upsilon / K-1) \quad, \quad M=3.180 \frac{\stackrel{\operatorname{sen} m}{K},}{K},
$$

formole adatte per valori di $K$ compresi tra 1 e 2,5-3, nel $2^{\circ}$ caso.

Dal valore di $M$ si deve ricavare quello di $C$; per $L=1$ Henry, $\omega=\pi .10^{3}, M=10^{3}(\pi-318,31 / C), M$ espresso in Ohm, $C$ in $m \mu F$.

Il calcolo riesce più spedito, (e sufficientemente approssimato), se si costruisce la curva: $M=M(C)$ per un numero sufficiente di ralori di $C$.

Ma in luogo del calcolo diretto mediante le formole che danno $\boldsymbol{X}+\Delta \boldsymbol{X}, \boldsymbol{M}$, si puó eseguire la determinazione degli stessi elementi per via puramente grafica, osservando cbe dette formole sono assolutamente simili a quelle che stanno alla base dell'impiego del compensatore. Si potranno dunque costruire nomogrammi di coordinate cartesiane ortogonali col solito criterio: in ascisse i valori di $M$ corrispondenti ai diversi valori di $C$ (e si quoteranno le ascisse con i valori stessi di $C$, in ordinate i diversi valori di $X+\Delta X$, agriunti a quelli di $R$.

Poi si tracceranno i diversi cerchi di rapporto, col centro nell'origine e raggi uguali a: $(R+X) / K$.

Infine si può aggiungere una graduazione goniometrica (limitata a raggi di $5^{c}$ in $5^{\circ}$ ) per rendere più spedite le letture occorrenti in queste determinazioni.

5. Descrizione e dispositivi degli apparecchi sul campo sperimentale. - Prima di passare in rassegna $i$ tipi di induttori impiegati (chiuso, aperto simmetrico, asimmetrico ecc.), completamente isolati dal suolo o no, dò una sommaria descrizione degli apparecchi impiegati (la maggior parte dei quali forniti temporaneamente da Istituti scientifici e Enti costruttori di apparecchi elettrici): 
1) generatore a diapason $\bmod .1649$; frequenza $500 \mathrm{~Hz}$; impedenza di uscita $250 \mathrm{Olım}$; potenza d'uscita $25 \mathrm{~mW}$; alimentazione con c.c. per mezzo di un survoltore di cui alla lettera 2);

2) survoltore mod. AC14; tensione anodica fornita al generatore a diapson a regime 270 Volt, con e. di $42 \mathrm{~mA}$; tensione di alimentazione $6 \mathrm{~V}$; corrente assorbita 8,2 Amp.;

3) amplificatore mod. D30P collegato all'uscita del generatore a diapson per l'amplificazione del segnale fornito dal medesimo; impedenza d'uscita $250 \mathrm{Ol} \mathrm{m}$; potenza d'uscita 1,7 Watt indistorti; alimentazione con c.c. fornita da un survoltore di cui a lettera (4);

4) survolatore mod. AC14; tensione anodica generata per l'alimentazione dell'amplificatore D30P a regime 230 Volt; con c. di 54 $\mathbf{m A}$; tensione di alimentazione $6 \mathrm{Volt}$; corrente assorbita a regime 10,5 Amp;

5) hatteria $5+5$ accumulatori al $F_{6}-N_{1}$ collegati in parallelo; capacita $70 \mathrm{Al}$; tensione $6 \mathrm{Volt}$; corrente erogata (alimentazione degli apparecchi di cui a lettera 1), 2), 3), 4); 18,7 Amp;

6) bohina esploratrice $I$, su rocchetto di legno; diametro medio avvolgimento $18 \mathrm{~cm} ; 2600$ spire di filo di rame da $\phi=0,3 \mathrm{~mm}$ smaltato; induttanza 1,5 Henry; resistenza a $20^{\circ} \mathrm{C}, 312$ Olım: capacità distribuita $140 \mathrm{pF}$.

La holina, provista di goniometro è montata su un sostegno di legno, tale da consentire qualsiasi orientamento poliassiale.

Modelli ancor più ridotti (es.: qualche cm di diametro), dovendo possedere un'autoinduzione apprezzabile, occorre che abbiano molti avvolgrimenti finissimi, il che comporta un enorme aumento della resisten. za olımica, quindi un " coefficiente di merito", bassissimo, con conse. guenti perdite di nitidezza nella risonanza e nella sensibilità. Per poter apprezzare allora piccole variazioni di $K$ e if, occorre apportare forti variazioni di $R_{.}$e $\tilde{C}$ nel circuito del compensatore, con sensibilità così piccola da rendere pressocché impossibile ogni sicura determinazione.

Le dimensioni delle hobine devono essere in scala con quelle del modello di conduttore interrato da rilevare, e dei campi m. secondari provocati dalle reazioni e.m. del conduttore: d'altra parte bobine troppo grandi abluracerebhero un valore medio di flusso n. risultante tale che in esso si annullano o si confondono le rapide variazioni locali.

7) quatretto di manovra del circuito $I$, montato sull'incastellatura di logno della hohina: 
8) bobina $I I$ uguale alla precedente, di cui a lettera (6);

9) cassetta di manovra del circuito $I I$, che deve rimanere in prossimità della bobina compensatrice $I I$, (con capacità di risonanza), per introdurre il meno possibile, a causa del cavo di collegamento, capacità distribuite, oltremodo dannose;

10) amplificatore speciale a 3 valvole sintonizzato sulla frequenza di $500 \mathrm{~Hz}$, per l'amplificazione del segnale risultante nel compeneatore: amplificazione a $500 \mathrm{~Hz}$ intorno al valore 17000 (85 db.);

11) misuratore d'uscita con portata: 3 - 15 - 30 - 75 - 150 Volt, impedenza costante $40.000 \mathrm{Ohm}$;

12) cuffia telefonica $4000 \Omega$. Come previsto quale istrumento di zero venne usato o un telefono alimentato attraverso ad un amplifi-

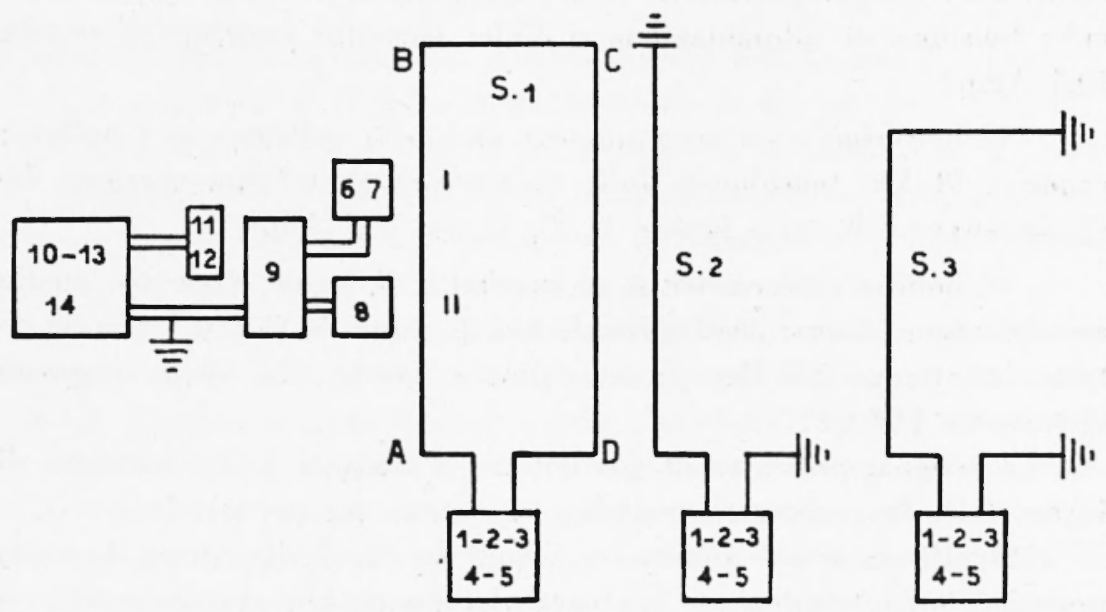

Fig. 9

catore-filtro con elevato fattore d'amplificazione, o uno strumento a lettura diretta, con varie sensibilità, ad impedenza costante, il quale facilita le prime approssimazioni nella ricerca dell'opposizione;

13) batteria di accumulatori per l'alimentazione dell'amplificatore di cui alla lettera (10); corrente erogata a regime 4 Amp;

14) survoltore mod. ACl4 per l'alimentazione anodica dell'amplificatore sintonizzato e alimentato a sua volta dalla precedente batteria di accumulatori; tensione anodica generata a regime $280 \mathrm{Volt}$, corrente $18 \mathrm{~mA}$.

L'alimentazione dell'apparecchiatura fatta con $i$ survoltori, alimentati a loro volta da 2 batterie di accumulatori, offre l'inconveniente che dopo poche ore di funzionamento le batterie si scaricano. Passando ora alla disposizione degli apparecchi e circuiti sul 
campo, riporto gli schemi principali (alcuni ne ho omessi, non apportando sostanzialmente nulla di nuovo): essi si riducono, in fondo, a tre schemi d'induttori: il $1^{\circ}$ (S.l) induttore puramente indultivo; il $2^{\circ}$ induttore galvanico-induttivo asimmetrico, tale che riducendo il tratto della presa di terra $D$ si riduce ad un induttore rettilineo; il $3^{\circ}$ galvanico-induttivo simmetrico (Fig. 9).

Lo schema: S.l, consta di un cappio-induttore, cavo teso $A B$ (lungo $\mathbf{m}$. 48 circa) a $\mathbf{m} 0,50$ sollevato dal suolo, completamente isolato dal terreno: la chiusura ha $B C=A D$ $=m 19$ circa. Ponendo la bobina $\mathrm{I}$ nell'interno del rettangolo alla distanza di $1 \mathrm{~m}$ dal filo teso $A B$, e la bobina II esternamente al cappio, alla distanza pure di $1 \mathrm{~m}$ dal tratio $A B$, si legre sul compensatore un rapporto: $K=450 / 400=1,125$, invece di uno ( $\varepsilon \%=+12,5 \%)$.

L'influenza del cavo di ritorno $C D$, anche alla distanza di $19 \mathrm{~m}$,

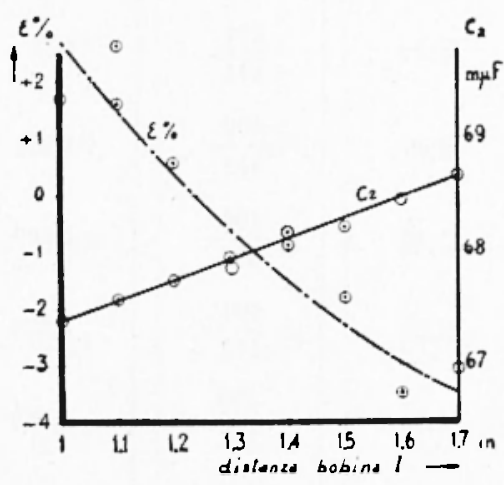

Fig. 10 da $A B$, permane notevole; infatti ponendo entrambe le bohine esternamente al cappio, alla distanza di 1 nı si legge il rapporto: $395 / 400=0,9775 \quad(\varepsilon \%=-2,25)$.

Per tentare d'eliminare l'inconveniente si sperimentò con $\mathrm{S} .2$ in cui l'estremo $B$ del filo teso va a terra, e cosi l'estremo $D$. In queste condizioni, con la bobina II fissa, alla distanza di m 1 dal filo teso,

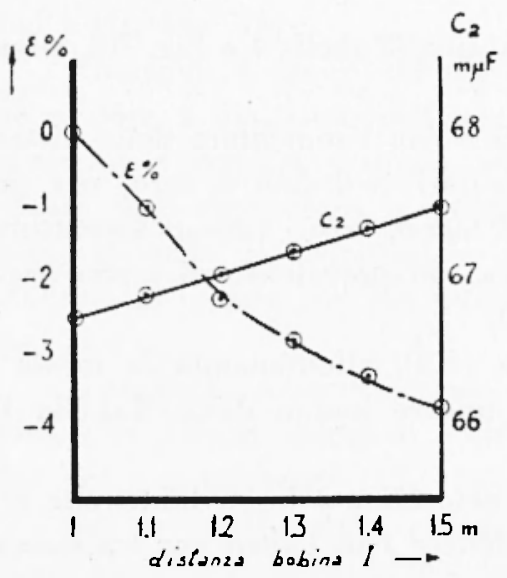

Fig. 11

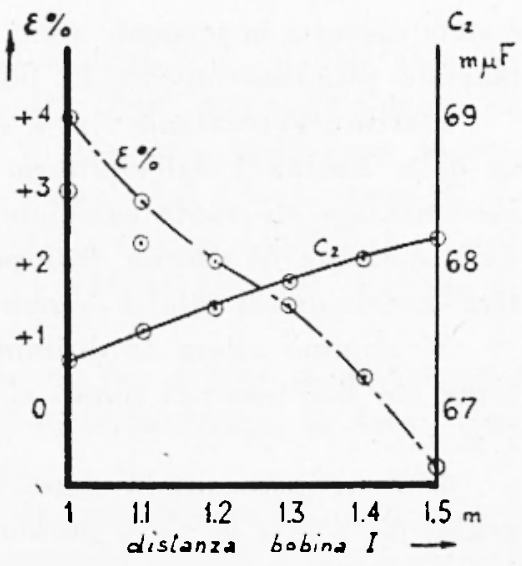

Fig. 12 
Tabelia I

\begin{tabular}{|c|c|c|c|c|c|}
\hline $\begin{array}{c}\text { Bob I } \\
\text { distanza } \\
\mathbf{m}\end{array}$ & $\begin{array}{l}\mathbf{R}+\mathbf{R}_{\mathbf{t}} \\
\mathbf{R}+\mathbf{R}=\end{array}$ & $\underset{\text { teorico }}{\mathrm{K}}$ & $\underset{\text { letto }}{\mathrm{K}^{\prime}}$ & $\varepsilon \%$ & $\begin{array}{c}\mathrm{C}_{\mathbf{g}} \\
\mathrm{m} / \mathrm{uF}\end{array}$ \\
\hline 1 & $\frac{400}{392}$ & 1 & 1,017 & $+1,7$ & 67,40 \\
\hline 1 & $\begin{array}{l}624 \\
614\end{array}$ & 1 & 1,013 & $+1,3$ & 67,40 \\
\hline 1,05 & $\frac{400}{411}$ & 0,952 & 0,977 & $+2,03$ & 67,48 \\
\hline 1,10 & $\begin{array}{l}400 \\
433\end{array}$ & 0,909 & 0,924 & $+1,05$ & 67,58 \\
\hline 1,20 & $\begin{array}{l}400 \\
478\end{array}$ & 0,833 & 0,838 & $+0,60$ & 67,75 \\
\hline 1.30 & $\begin{array}{l}400 \\
528\end{array}$ & 0,769 & 0,759 & $-1,30$ & 67,96 \\
\hline 1,40 & $\begin{array}{l}400 \\
564\end{array}$ & 0,714 & 0,709 & $-0,7$ & 68,05 \\
\hline 1,50 & $\frac{400}{612}$ & 0,666 & 0,654 & $-1,8$ & 68,22 \\
\hline 1.60 & $\begin{array}{l}400 \\
662\end{array}$ & 0,625 & 0,603 & $-3,5$ & 68,46 \\
\hline 1.70 & $\frac{400}{702}$ & 0,588 & 0,570 & $-3,06$ & 68,68 \\
\hline
\end{tabular}

è stata eseguita la seguente serie di misure (Tabella I e Fig. 10), allontanando progressivamente la bobina $\mathbf{I}$ :

L'errore percentuale: $\varepsilon \%$ decresce con l'aumentare della distanza della bohina I dall'emittore, da valori $>0$ fino a zero, per poi incrementarsi di nuovo cambiando di segno, errori causati soprattutto dalla corrente di ritorno disimmetrica con prevalenza di segno negativo avvicinandosi alla diagonale $B D$.

Si eliminò allora la disimmetria (S.3), allontanando la messa a terra del filo teso nel punto $C$. Le misure hanno dato (Tabella II, Fig. 11).

L'errore percenutale segue una determinata legge tanto cbe i 2 profili di $\varepsilon \%$ e di $C$. si possono ottenere l'un l'altro per traslazione lungo le ordinate. 
TABella II

\begin{tabular}{|c|c|c|c|c|c|}
\hline $\begin{array}{c}\text { Bob I } \\
\text { distanza } \\
\mathbf{m}\end{array}$ & $\frac{R+R_{1}}{R+R_{2}}$ & $\underset{\text { teorico }}{\mathrm{K}}$ & $\underset{\text { letto }}{\mathbf{K}^{\prime}}$ & \&. \% & $\begin{array}{c}\mathrm{C}_{2} \\
\mathrm{~m} / \mathrm{uF}\end{array}$ \\
\hline 1 & $\frac{400}{400}$ & 1 & 1 & - & 66,72 \\
\hline 1 & $\frac{624}{627}-$ & 1 & 0,999 & $-0,1$ & 66,41 \\
\hline 1,10 & $\frac{400}{445}$ & 0,909 & 0,900 & $-1,01$ & 66,90 \\
\hline 1,10 & $\frac{624}{695}$ & 0,909 & 0,900 & $-1,01$ & 66,46 \\
\hline 1,20 & $\frac{400}{492}$ & 0,833 & 0,814 & $-2,28$ & 67,03 \\
\hline 1,30 & $\frac{400}{537}$ & 0,769 & 0,747 & $-2,86$ & 67,19 \\
\hline 1,40 & $\frac{400}{580}$ & 0,714 & 0,690 & $-3,36$ & 67,32 \\
\hline 1,50 & $\begin{array}{l}400 \\
624\end{array}$ & 0,666 & 0,641 & $-3,76$ & 67,47 \\
\hline
\end{tabular}

Lo schema S.4 ripete il cappio puramente induttivo, salvo l'allontanamento in $D C$ del complesso 1-2-3-4-5 dall'emittore $A B$ (Tabella III, Fig. 12):

Abolendo i complessi survoltori-accumulatori, riallacciando nel generatore a diapason, nell'amplificatore D30P nell'amplificatore sintonizzato i circuiti d'alimentazione in c.a., già predisposti internamente negli apparecchi, e collegando i medesimi ad una presa di corrente normale di distribuzione d'energia elettrica a 160 Volt, si sono ripetute le misure con S.3 ottenendo i seguenti risultati (Tabella IV, fig. 13):

Il seguente schema è stato adoperato per misure di differenze di fase, con diversi valori di resistenza e di capacità, calcolate e lette poi nel compensatore (Fig. 14).

Per la verifica delle condizioni di risonanza con l'oscillografo a raggi catodici (accennato a $\$ 3$ ) il controllo è stato eseguito introdu- 
Tabella III

\begin{tabular}{|c|c|c|c|c|c|}
\hline $\begin{array}{c}\text { Bob I } \\
\text { distanza } \\
\text { m }\end{array}$ & $\frac{\mathbf{R}+\mathbf{R}_{\mathbf{1}}}{\mathbf{R}+\mathbf{R}_{\mathbf{2}}}$ & $\underset{\text { teorico }}{\mathrm{K}}$ & $\underset{\text { letto }}{K^{\prime}}$ & $\varepsilon \%$ & $\begin{array}{c}\mathrm{C}_{2} \\
\mathrm{~m} / \mathrm{uF}\end{array}$ \\
\hline 1 & $\frac{400}{334}$ & 1 & 1,04 & +4 & 67,39 \\
\hline 1 & $\frac{604}{606}$ & 1 & 1,03 & +3 & 67,37 \\
\hline 1,10 & $\begin{array}{l}400 \\
429\end{array}$ & 0,909 & 0,935 & $+2,86$ & 67,56 \\
\hline 1,10 & $\frac{624}{674}$ & 0,909 & 0,930 & $+2,3$ & 67,56 \\
\hline 1,20 & $\begin{array}{l}400 \\
470\end{array}$ & 0,833 & 0,850 & $+2,04$ & 67,7 \\
\hline 1,30 & $\begin{array}{l}400 \\
514\end{array}$ & 0,769 & 0,780 & $+1,43$ & 67,91 \\
\hline 1,40 & $\frac{400}{558}$ & 0,714 & 0,717 & $+0,12$ & 68,118 \\
\hline 1,50 & $\begin{array}{r}400 \\
604\end{array}$ & 0,667 & 0,662 & $-0,75$ & 68,16 \\
\hline
\end{tabular}

cendo volta per volta nel circuito in prova una bobina tipo "Curtiss" particolarmente studiata per ridurre l'induttanza e lat capacità distribuita dell'avvolgimento.

All'asse $x$ dell'oscillografo si è collegato la caduta di tensione ai capi della bobina "Curtiss" e all'asse $y$ la tensione di uscita dol. l'amplificatore. Se nel tubo si disegna un'ellisse (in genere molto schiacciata), ciò dimostra che la corrente nel circuito non $\dot{e}$ in fase con la tensione applicata. Il rifasamento si può ottenere, per i circuiti delle bobine di esplorazione, agendo sui condensatori di correzione, già predisposti nei circuiti di compensazione.

Risultati ottenuti con modello pane di $\mathrm{P}_{\mathrm{b}}$ interrato. - Tenendo fissa la bolina orizzontale $(500 \mathrm{~Hz}, 150 \mathrm{~mA})$ II, alla distanza di m 2 dall'emittore, cavo teso, a m 0,21 di altezza dal terreno discosta da un blocco di piombo (conduttore perturbante posto nel suolo, parallelepipedo di dimensioni: $m 0,64 \times 0,11 \times 0,055$, peso $\mathrm{kg} 44,5$ ), gli apparecchi di- 
sposti secondo lo schema S.3, la bohina mobile I allontanandosi progressivamente dall'induttore complanare di cui si vede traccia in Fig. 15 fino ad oltrepassare il piombo, ho ottenuto risultati di misure riassunti nella Tabella $\mathrm{V}$ e nel grafico stesso Fig. 15 (diagrammi relativi alle misure eseguite su modello di piombo con bohine schermate).

I comportamenti relativi di $K$ e $\varphi$, che, come si osserva nella Fig. 15, rendono possibile la localizzazione accurata del conduttore perturbante, permettono altresì la determinazione di una scala delle indicazioni, a seconda l'entità della conduttività elettrica dei con-

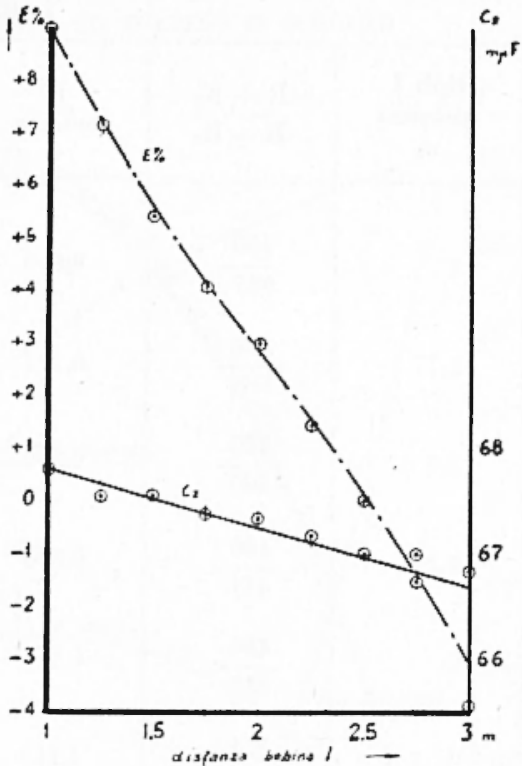

Fig. 13 duttori stessi.

A secondo i valori relativi $\Delta p, K$, si può risalire in altri termini alla precisazione della conduttività del conduttore sepolto nel suolo, e quindi, (equivalenze a parte), alla sua consistenza mineraria.

Ė ben noto ai prospettori che non solo i problemi ubicazionali, di tonnellaggio, interessano la ricerca geofisica dei minerali nel soltosuolo, spesso questi passano in seconda linea di fronte a quello della determinazione della natura fisica dei minerali stessi, a meno che non

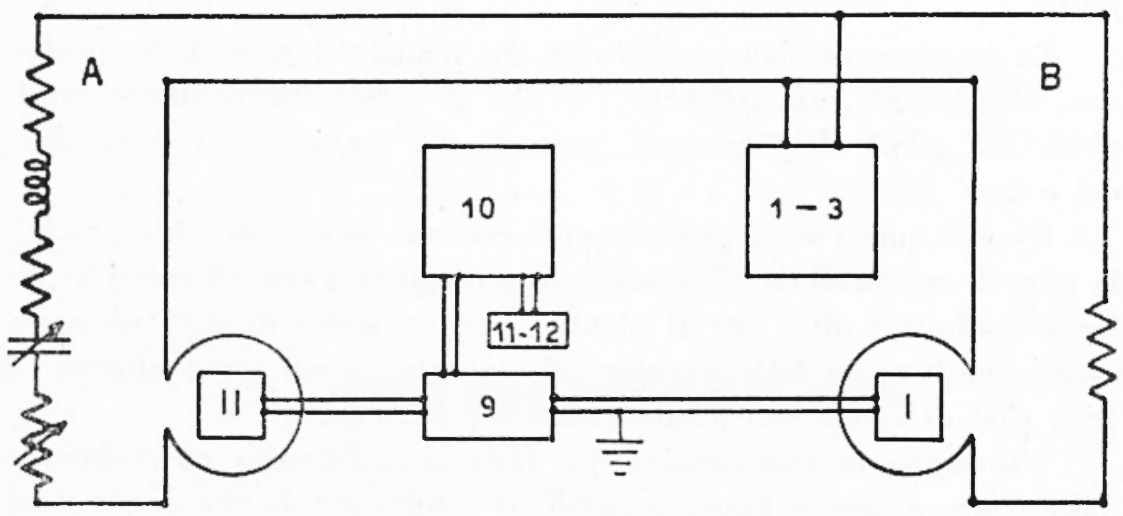

Fig. 14 
Tabella IV

\begin{tabular}{|c|c|c|c|c|c|}
\hline $\begin{array}{c}\text { Bob I } \\
\text { distanza } \\
m\end{array}$ & $\begin{array}{l}\mathbf{R}+\mathbf{R}_{\mathbf{L}} \\
\mathbf{R}+\mathbf{R} .\end{array}$ & $\underset{\text { teorico }}{\mathrm{K}}$ & $\underset{\text { letto }}{\mathrm{K}^{\prime}}$ & $\varepsilon \%$ & $\begin{array}{c}\mathrm{C}_{2} \\
\mathrm{~m} / \mathrm{uF}\end{array}$ \\
\hline 3 & $\frac{400}{625}$ & 0,666 & 0,640 & $-3,9$ & 66,84 \\
\hline 2,75 & $\frac{400}{556}$ & 0,727 & 0,720 & $一 0,96$ & 66,75 \\
\hline 2,5 & $\frac{400}{500}$ & 0,800 & 0,800 & 0 & 67 \\
\hline 2,25 & $\begin{array}{l}400 \\
4.4\end{array}$ & 0,888 & 0,901 & $+1,46$ & 67,21 \\
\hline 2 & $\begin{array}{l}400 \\
388\end{array}$ & $1-$ & 1,03 & +3 & 67,36 \\
\hline 1,75 & $\begin{array}{r}624 \\
522\end{array}$ & 1,143 & 1,190 & $+4,1$ & 67,4 \\
\hline 1,50 & $\begin{array}{l}624 \\
444\end{array}$ & 1,333 & 1,405 & $+5,4$ & 67,56 \\
\hline 1,25 & $\frac{936}{546}$ & 1,600 & 1,715 & $+7,2$ & 67,56 \\
\hline 1 & $\begin{array}{l}936 \\
428\end{array}$ & $2-$ & 2,180 & +9 & 67,80 \\
\hline
\end{tabular}

intervengano concomitanti indicazioni geologiche di una certa probabilità.

La sperimentazione geoelettrica, da tempo, ci mostra, in proposito, correlazioni caratteristiche (ai fini di poter risalire alle conduttività dei corpi elettricamente perturbanti) tra isofasi e isodiname dei c.e.m.

Usando questi stessi procedimenti, con una tecnica di misura (e.m.) la più efficace possibile (Bicaram), sto proseguendo con gli esami di diversi conduttori oltre che il piombo, mentre spero di dare una griustificazione teorica delle correlazioni scoperte, di cui sopra almeno in certi casi di conduttori geometricamente ben definibili.

L'indagine modellometrica qui fatta si differenzia notevolmente dalle scarse e incerte finora apparse: il conduttore da rintracciare col Bicaram non è un cosidetto "asse elettrico" (cavo percorso da c.e. 
niangrammi relativi alle misure eseguite su modello di piombo

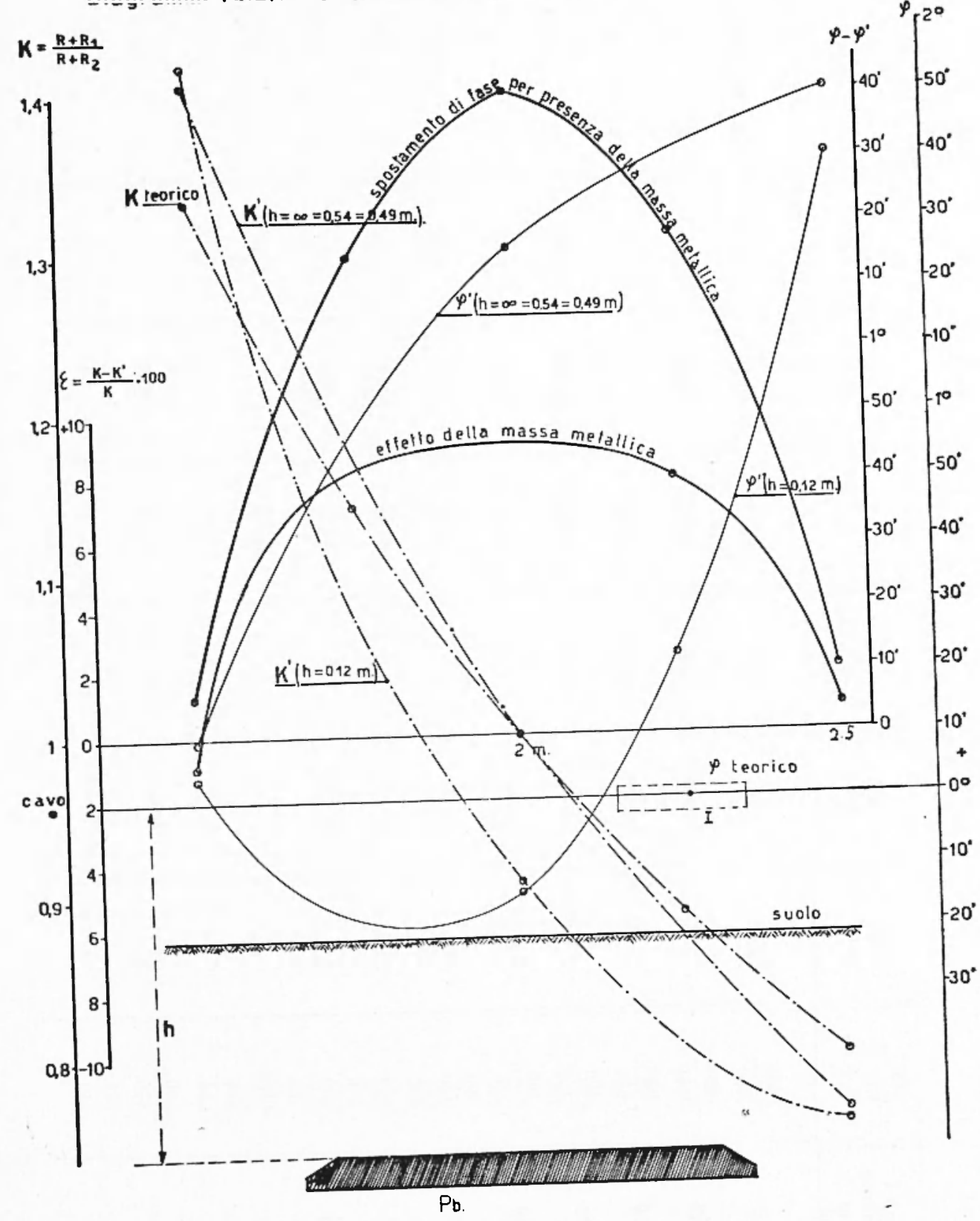

Fig. 15 


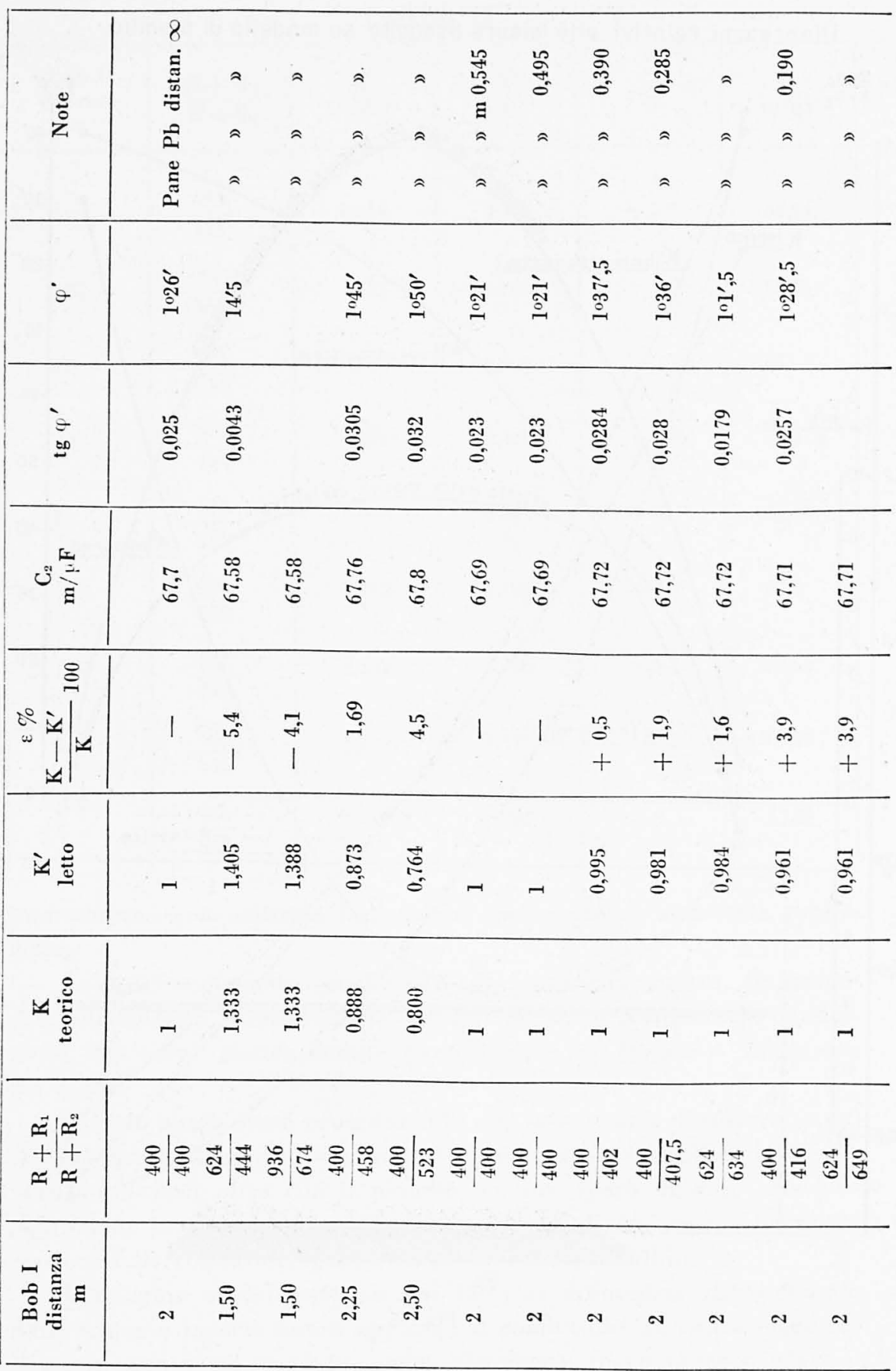




\begin{tabular}{|c|c|c|c|c|c|c|c|c|c|c|c|}
\hline ¿ัँ & 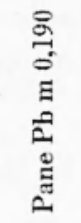 & $\begin{array}{l}\approx \\
\approx \\
=\end{array}$ & $\begin{array}{l}a \\
= \\
=\end{array}$ & $\begin{array}{l}a \\
\approx \\
=\end{array}$ & $\begin{array}{l}\approx \\
\approx \\
\approx\end{array}$ & $\begin{array}{l}\stackrel{0}{7} \\
0 \\
0 \\
\therefore\end{array}$ & $\begin{array}{l}\approx \\
\approx \\
=\end{array}$ & $\begin{array}{l}\approx \\
\approx \\
\therefore\end{array}$ & $\begin{array}{l}2 \\
\approx \\
\therefore\end{array}$ & $\begin{array}{l}\approx \\
\approx \\
\approx\end{array}$ & $\begin{array}{l}\curvearrowright \\
\approx \\
\approx\end{array}$ \\
\hline$\theta$ & 望 & $\stackrel{30}{=}$ & is & 官 & ㅇ & $\frac{n}{12}$ & & $\stackrel{13}{10}$ & $\begin{array}{l}\text { in } \\
+\end{array}$ & มิ่ & 㐔 \\
\hline 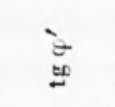 & $\begin{array}{c}\stackrel{0}{\circ} \\
\stackrel{0}{0}\end{array}$ & 渵 & $\begin{array}{l}\infty \\
\stackrel{\infty}{0} \\
\stackrel{0}{0}\end{array}$ & 梁 & 管 & 总 & & $\begin{array}{l}20 \\
0 \\
0 \\
0\end{array}$ & "ే & ஜूँ & ఫิ \\
\hline ง & $\begin{array}{l}8 \\
6 \\
6\end{array}$ & $\begin{array}{l}\infty \\
\substack{0 \\
6 \\
6}\end{array}$ & $\frac{9}{6}$ & 8 & : & 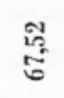 & $\begin{array}{l}\text { 웅 } \\
6 \\
6\end{array}$ & $\begin{array}{l}\text { 茴 } \\
\text { - }\end{array}$ & \begin{tabular}{l}
0 \\
20 \\
\multirow{6}{*}{}
\end{tabular} & : & 息 \\
\hline 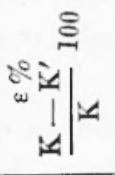 & 응 & $\begin{array}{l}\mathscr{0} \\
\text { if } \\
\text { | }\end{array}$ & $\begin{array}{l}\stackrel{0}{\leftarrow} \\
+\end{array}$ & $\begin{array}{l}\text { है } \\
+\end{array}$ & $\begin{array}{l}\text { 璁 } \\
+\end{array}$ & 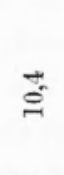 & $\Rightarrow$ & $\stackrel{2}{0}$ & $\begin{array}{l}\overrightarrow{0} \\
1\end{array}$ & $\begin{array}{l}\stackrel{\circ}{0} \\
+\end{array}$ & $\begin{array}{l}\infty \\
\text { in } \\
+\end{array}$ \\
\hline 江惫 & $\stackrel{\overrightarrow{9}}{=}$ & $\exists$ & $\begin{array}{l}\overrightarrow{0} \\
0 \\
0\end{array}$ & 융 & $\underset{0}{\stackrel{0}{\circ}}$ & $\begin{array}{l}\mathscr{0} \\
\text { : } \\
\text { o }\end{array}$ & $\begin{array}{l}\text { : } \\
\text { हे }\end{array}$ & $\underset{\overbrace =}{\stackrel{N}{G}}$ & $\stackrel{\infty}{\underset{\sim}{\rightleftarrows}}$ & $\begin{array}{l}\text { ఇ్ } \\
\text { ळ }\end{array}$ & 窎 \\
\hline 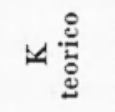 & $\underset{ت}{\stackrel{\Xi}{二}}$ & ๙ึ? & $\begin{array}{l}80 \\
00 \\
0 \\
0\end{array}$ & $\begin{array}{l}\stackrel{\circ}{\circ} \\
\circ\end{array}$ & $\begin{array}{c}\mathscr{E} \\
\stackrel{\infty}{0} \\
\delta\end{array}$ & - & $r$ & $\underset{\mathfrak{I}}{\stackrel{\mathcal{F}}{\leftrightarrows}}$ & 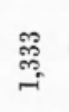 & $\begin{array}{l}\infty \\
\stackrel{0}{0} \\
0\end{array}$ & 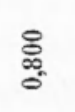 \\
\hline 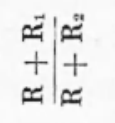 & 헝| & ్ㅐㅇㅝㅝ & 岁| & 웡 & ำำ & 잏 & ్ㅓㅇ|ळ & त్ํ요 & 항|염 & $\stackrel{8}{\not}$ & ㅇㅣㅎㅀㅠ \\
\hline ص䒿 & $\stackrel{9}{\stackrel{9}{2}}$ & 铵 & 영 & 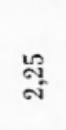 & 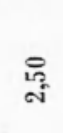 & N & N & $\stackrel{0}{2}$ & 옥 & สู สิ & 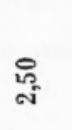 \\
\hline
\end{tabular}


senza alcun riferimento col suolo), esso è invece un ot timo conduttore massivo $\left(P_{\mathrm{b}}\right)$ immerso nel cattivo o medio conduttore suolo incassante.

Le sue reazioni e.m. specifiche, $K$ e $\uparrow$, sono ottenute infatti come differenze :

$$
K\left(P_{\mathrm{b}}+\text { suolo }\right)-K(\text { suolo }) \equiv K^{\prime}(h=\mathrm{m} 0,12)-K^{\prime}(h=\boldsymbol{\alpha})
$$

dove $h$ è la distanza verticale (o profondita) variabile tra la bobina e il piombo interrato.

La semplice idea di sostituire ad una tesata di cavetto metallico nell'aria (ottima per semplificare le indagini modellometriche di massima) un blocco di piombo interrato puó non esser stata attuata in precedenza per più motivi: la sottovalutazione delle reazioni e.m. dei suoli omogenei incassanti, (il cui importante contributo fu studiato soprattutto da A. Graf), e la difficoltà di realizzazioni modellometriche (strumenti, grandezze fisiche, ecc.) nel senso voluto.

La fig. 15 con i profili $K$, sia pure molto estrapolati, evidenzia un andamento di $K$ teorico prossimo al $K^{\prime}(\infty)$ del suolo omogeneo, con scostamenti seguenti una determinata legge, (allontanandosi la bobina rilevatrice I dall'emittore), attenuantesi a distanza.

Si deve tener presente che l'esperienze vennero condotte, (perché non pronti), senza l'inserimento nel Bicaram dei necessari trasformatori d'isolamento, quindi in condizioni non ottime.

Caratteristici i profili d'anomalia $K^{\prime}, \varphi^{\prime},(h=0,12 \mathrm{~m})$, in relazione all'ubicazione e morfologia del conduttore e l'effetto $K^{\prime}$, $\varphi^{\prime}$ del terreno $(h=\infty)$. Si comprende subito, esaminando la figura, come una copertura conduttiva, (caso frequente alle nostre latitudini), possa provocare una sola coppia $K^{\prime}, \varphi^{\prime}$, come se il conduttore $P_{\mathrm{b}}$, (anche a piccola profondità), non esistesse, riducendosi a zero l'area compresa tra i 2 profili $K^{\prime}(\propto), K^{\prime}(0,12)$. Le diagrafie della Fig. 15, danno in definitiva il modo di constatare risultati sperimentali nettamente positivi, distinti e convergenti per l'individuazione di masse conduttrici sepolte, irraggiungibile, nelle stesse condizioni, con altri compensatori. Si al massimo possibile perfezionamento strumentale dei geocompensatori a c.a. a bobine bilanciate, l'impiego dei quali tuttavia rimarri positivo solo se essi, (specie in condizioni difficili di rilevamento ad es. di solfuri misti sardi di $P_{\mathrm{h}}$ e $Z_{n}$ ) saranno inseriti nei dispositivi tribobinici (Trib) da me introdotte (geoinduttore, schermi di c.m. primario, detector geocompensatore Bicaram).

Cagliari - Istituto di Geofisica Mineraria - Novembre 1952. 


\section{RIASSUNTO}

L'A., nella "Premessa", ricorda $i$ suoi precedenti Studi che hanno portato alla realizzazione d'un nuovo compensatore a ca. denominato Bicaram.

Con la presente Nota completa una serie d'esperienze a scala ridotta, permettenti ulteriori affinamenti strumentali, con copioso materiale di sperimentazione.

I risultati ottenuti con esperienze su modello di $\mathrm{P}_{\mathrm{b}}$ interrato, riassunti in apposito grafico, mostrano profili di campo e.m. e di fase cospiranti a localizzare il buon conduttore, e definirne il grado di condutività; rivelano la notevole influenza dei suoli incassanti, delle coperture conduttive di mascheramento.

Riferendosi a precedenti suoi lavori sull'argomento, l'A. accenna al razionale e più redditizio impiego di tali compensatori in genere, nolle zone di eliminazione del c.e.m. primario, ottenute con diversi procedimenti dell'A. stesso.

\section{SUMMARY}

The author, in "Premessa" summarizes his previous studies which have lead to a new type of $A C$ compensator called Bicaram.

With the present note, the description of a series of experiments on a reduced scale is completed; giving further instrumental refinements and including a large amount of experimental data.

The results obtained from experiments using buried $P_{\mathrm{t}}$ models are summarized in an appropriate graph which shours profiles of the o.m. field and the phase which together serve to locate a good conductor and to define for it the degree of conductivity, revealing the notable influence of packed soils and the masking effect of conducting layers.

Referring to his previous uorks on this question, the author calls attention to the possibility of using this compensutor for the elimina. tion of the primary e.m. field, as has been done in various uays by the author himself. 


\section{BIBLIOGRAFIA}

(1) Arnalbo Belluigr: Accoppiamenti resistivi di bobine induttive nella prospezione elettrica del sottosuolo. La Ricerca Scientifica, Roma 1942, pag. 770.

A. B.: Accoppiamenti resistivi di bobine induttive ecc. (Teoria, Esperimenti di Laboratorio). Boll. Uff. Geologico d'Italia, Roma 1943, pag. 13.

A. B.: Impiego del compensatore a c. a., a bobine bilanciate, nelle misure in scala ridotta. Rend. del Seminario della Facoltà di Scienze, Cagliari, 1946.

A. B.: Bicaram. (Nuova apparecchiatura e $\mathrm{m}$, a sensibilità variabile). Boll. Uff. geologico d'Italia, Roma 1947, pag. 17 .

A. B.: Sulle prospezioni elettromagnetiche di alcune tipiche giaciture di solfuri misti in Sardegna, ecc. Atti del Congresso Minerario Italiano, 1948.

(-) A. B.: La misura diretta dei c.e.m secondari dei suoli; il Mind, il Trib. Rend. Seminario delle Facoltà di Scienze, Cagliari 1946.

A. B.: Fondamenti di una nuova prospezione geoelettrica del 1947. Pubbl. della Iracolta d'Ingegneria di Cagliari.

A. B.: L'impiego della geoelettrica in Miniera. Experientia Basel 19.18.

A. B.: Problemi fondamentali concernenti limpiego dei metodi indutivi ecc. Rendiconti Seminario Facoltà di Scienze, Cagliari 1948.

A. B.: Su una nuova prassi geoelettrica induttiva per ricerche di conduttori nel sottosuolo. Boll. Servizio Geologico d'Italia, 1947.

(3) Geyer W.: Die Anwendung des Komplexen Wechselstromkompensators bei geol. Untersuchungen. Archiv fiir Elektr. 1929, H. “Messungen mit dem Schleifdraht-Wechselstrokomp. " E.T.Z, 1930.

Giaro Joserh A.: Compensateur double à courant alternatif. Leemann S.A, Zurich 1949.

Barbagelata Angelo: Misure elettriche. Libreria Editrice Politecnica, Milano, volume II pag. 180 e seg. 